The Astrophysical Journal: Accepted 11 Nov. 2010

\title{
Explosive Outflows Powered by the Decay of Non-Hierarchical Multiple Systems of Massive Stars: Orion $\mathrm{BN} / \mathrm{KL}$
}

\author{
John Bally \\ Center for Astrophysics and Space Astronomy, \\ University of Colorado, Boulder, CO 80309 \\ John.Bally@colorado. edu \\ Nathaniel J. Cunningham \\ Department of Physics and Astronomy, University of Nebraska-Lincoln \\ 116 Brace Laboratory, Lincoln, NE 68588-0111 \\ ncunningham2@unl.edu \\ Nickolas Moeckel \\ Institute of Astronomy, University of Cambridge \\ Madingley Road, Cambridge CBз OHA, England \\ moeckel@ast.cam.ac.uk \\ Michael G. Burton \\ School of Physics, University of New South Wales \\ Sydney, Australia, NSW 2052 \\ mgb@phys . unsw . edu . au \\ Nathan Smith \\ Astronomy Department, Steward Observatory, University of Arizona, Tucson AZ \\ nathans@as . arizona.edu
}

Adam Frank 
Dept. of Physics and Astronomy, University of Rochester, Rochester, NY, 14627

afrank@pas.rochester .edu

Ake Nordlund

Astronomical Observatory and Theoretical Astrophysics Center, Juliane Maries Vej 30, DK-2100 Copenhagen, Denmark

aake@astro.ku.dk

\begin{abstract}
The explosive BN/KL outflow emerging from OMC1 behind the Orion Nebula may have been powered by the dynamical decay of a non-hierarchical multiple system $\sim 500$ years ago that ejected the massive stars I, BN, and source n, with velocities of about 10 to $30 \mathrm{~km} \mathrm{~s}^{-1}$. New proper motion measurements of $\mathrm{H}_{2}$ features show that within the errors of measurement, the outflow originated from the site of stellar ejection. Combined with published data, these measurements indicate an outflow age of $\sim 500$ years, similar to the time since stellar ejection. The total kinetic energy of the ejected stars and the outflow is about 2 to $6 \times$ $10^{47}$ ergs. It is proposed that the gravitational potential energy released by the formation of a short-period binary, most likely source I, resulted in stellar ejection and powered the outflow. A scenario is presented for the formation of a compact, non-hierarchical multiple star system, its decay into an ejected binary and two high-velocity stars, and launch of the outflow. Three mechanisms may have contributed to the explosion in the gas: (i) Unbinding of the circumcluster envelope following stellar ejection, (ii) disruption of circumstellar disks and high-speed expulsion of the resulting debris during the final stellar encounter, and (iii) the release of stored magnetic energy. Plausible proto-stellar disk end envelope properties can produce the observed outflow mass, velocity, and kinetic energy distributions. The ejected stars may have acquired new disks by fallback or Bondi-Hoyle accretion with axes roughly orthogonal to their velocities. The expulsion of gas and stars from OMC1 may have been driven by stellar interactions.
\end{abstract}

Subject headings: star formation: general — nebulae: individual (Orion Nebula, OMC1) 


\section{Introduction}

Massive stars are relatively rare and tend to form in distant, obscured, crowded and dynamic environments. The Orion Molecular Cloud 1 (OMC1) located immediately behind the Orion Nebula is the nearest site of ongoing massive star birth with a bolometric luminosity of about $10^{5} \mathrm{~L}_{\odot}$ (Gezari, Beckman, \& Werner 1998). OMC1 contains the Becklin-Neugebauer (BN) object (Becklin \& Neugebauer 1967; Scoville et al. 1983), the first massive young stellar object to be discovered in the infrared and the Kleinman-Low (KL) thermal-infrared nebula (Kleinmann \& Low 1967) which contains additional highly obscured massive stars. In addition to $\mathrm{BN}$, radio sources I and $\mathrm{n}$ are thought to be massive because they are detected at radio wavelengths (Menten \& Reid 1995). Recent sub-millimeter interferometry has revealed the presence of an additional protostellar candidate, SMA1 about $2^{\prime \prime}$ south-south west of radio source I (Beuther et al. 2006; Beuther \& Nissen 2008). Although this is the brightest source in the sub-mm, it has not been detected at other wavelengths.

The BN/KL region contains a spectacular, wide opening-angle, arcminute-scale outflow (Allen \& Burton 1993) traced by molecules such as $\mathrm{CO}$ and $\mathrm{NH}_{3}$ that exhibit broad $(>100$ $\mathrm{km} \mathrm{s}^{-1}$ ) emission line wings (Kwan \& Scoville 1976; Wiseman \& Ho 1996; Furuya \& Shinnaga 2009) and high-velocity $\mathrm{OH}, \mathrm{H}_{2} \mathrm{O}$, and $\mathrm{SiO}$ maser emission (Genzel et al. 1981; Greenhill et al. 1998). This bipolar outflow with a southeast (red-shifted) - northwest (blue-shifted) axis contains at least $8 \mathrm{M}_{\odot}$ of accelerated gas with a median velocity of about $20 \mathrm{~km} \mathrm{~s}^{-1}$. Interferometric $\mathrm{CO}$ images, $\mathrm{H}_{2} \mathrm{O}$ masers, and dense-gas tracers such as $\mathrm{SiO}$ reveal smaller ( $8^{\prime \prime}$ long) and younger ( $\sim 200$ year old) outflow along a northeast-southwest axis emerging from radio source I orthogonal to the arc-minute-scale CO outflow (Beuther \& Nissen 2008; Plambeck et al. 2009). The momentum and kinetic energy content of these flows is at least $160 \mathrm{M}_{\odot} \mathrm{km} \mathrm{s}^{-1}$ and $4 \times 10^{46} \mathrm{ergs}$ (Snell et al. 1983) to $4 \times 10^{47} \mathrm{ergs}$ (Kwan \& Scoville 1976) Zapata et al. (2009) presented a CO J $=2-1$ interferometric study and found a dynamic age of about 500 years for the larger OMC1 outflow. They noted its impulsive nature, that its structure is different from accretion-disk powered flows, and that it originated several arc-seconds north of the OMC1 hot-core.

While most protostellar outflows appear to be driven by jets or collimated winds (Reipurth \& Bally 2001; Beuther \& Shepherd 2005; Arce et al. 2007; Qiu et al. 2008), the BN/KL outflow is a wide-angle explosion. Near-infrared images reveal hundreds of individual bow shocks in the lines of [FeII] and $\mathrm{H}_{2}$ that emerge in nearly every direction (Allen \& Burton 1993; Kaifu 2000). The higher excitation species such as the $1.64 \mu \mathrm{m}$ line of [FeII] trace the bow shock tips (dubbed "bullets") while lower excitation tracers such as $\mathrm{H}_{2}$ trace the wakes or bow-shock skirts (dubbed "fingers"). A few of these shocks protrude from the molecular cloud and can be seen at visual wavelengths in [OI], [SII], [NII], and $\mathrm{H} \alpha$ (Axon \& Taylor 
1984; O'Dell et al. 1997). The highest velocity shock, HH $210\left(\sim 400 \mathrm{~km} \mathrm{~s}^{-1}\right)$ has been detected in X-rays (Grosso et al. 2005). Proper motions indicate a common age of about $10^{3}$ years or less for many features (Doi, O'Dell, \& Hartigan 2002), comparable to the 500 year age estimate of Zapata et al. (2009).

The three brightest radio-emitting stars in OMC1, sources $\mathrm{BN}$, I, and $\mathrm{n}$, have proper motions (motions in the plane of the sky) of 25, 15, and $26 \mathrm{~km} \mathrm{~s}^{-1}$ away from a region less than $500 \mathrm{AU}$ in diameter from which they were ejected about 500 years ago (Rodriguez et al. 2005; Gomez et al. 2005; 2008). Apparently, a non-hierarchical multiple star system containing at least 4 members experienced a dynamical interaction resulting either in the formation of a tight binary or possibly even a stellar merger (Bally \& Zinnecker 2005; Bally 2008; Zapata et al 2009) whose (negative) gravitational binding energy ejected these stars from the OMC1 core. With estimated stellar masses of 8 to 13,12 to 20 , and 2 to $3 \mathrm{M}_{\odot}$ for $\mathrm{BN}$, I, and $\mathrm{n}$ respectively, the kinetic energy of the ejected stars is 1 to $2 \times 10^{47}$ ergs (Rodriguez, et al., 2005; Gomez et al. 2005; 2008). Thus, the total kinetic energy of the ejected stars and the outflow is about 1.5 to $5.5 \times 10^{47}$ ergs. Assuming that the outflow has suffered some dissipation of its original kinetic energy as it interacted with its surroundings, a reasonable range of initial energies required to eject the stars and outflow is 2 to $6 \times 10^{47}$ ergs.

In this paper, new proper motion measurements of $\mathrm{H}_{2}$ are presented and combined with published data. The results indicate a 500 year age for the fastest ejecta in the OMC1 bullet and finger system and show that the point of origin of the flow coincides with the location of the ejected stars 500 years ago to within the measurement errors. Motivated by an apparent common event that ejected both the stars and the outflow, we present a plausible scenario for the recent evolution of the OMC1 region.

\section{Observations: Proper Motions of Features in the BN/KL Outflow}

New images of the BN/KL outflow were obtained on 21 November 2004 with Near Infrared Camera and Fabry-Perot Spectrometer (NICFPS) on the ARC $3.5 \mathrm{~m}$ telescope at Apache Point Observatory in New Mexico. NICFPS uses a $1024 \times 1024$ pixel Rockwell Hawaii 1-RG HgCdTe detector. The pixel scale of this instrument is $0.273^{\prime \prime}$ per pixel with a field of view $4.58^{\prime}$ on a side. Images with 300 second exposures were obtained in the 2.122 $\mu \mathrm{m} \mathrm{S}(1)$ line of $\mathrm{H}_{2}$ using a narrow-band filter (FWHM $=0.4 \%$ of the central wavelength). Separate sky frames were obtained using the same exposure time at a location $600^{\prime \prime}$ east. A set of 5 dithered images were obtained both on-source and on the sky position. A mediancombined set of unregistered, mode-subtracted sky frames were used to form a master sky- 
frame that was subtracted from individual images. The reduced images were corrected for optical distortions. Field stars were used to align the frames which were median-combined to produce the image shown in Figure 1. Atmospheric seeing produced 0." 8 . full-width at half-maximum stellar images. A full description of the data acquisition, the data reduction, and analysis procedures is given in Cunningham (2006).

Proper motions for individual features in the BN/KL outflow were determined by comparison of the 2004 epoch NICFPS image with $\mathrm{H}_{2}$ images taken on 13 September 1992 using the IRIS instrument at the Anglo-Australian Telescope (AAT) described by Allen \& Burton (1993), and the narrowband images taken on 11 and 13 January 1999 with the CISCO instrument on the Subaru telescope on Mauna Kea, Hawai'i (Kaifu et al. 2000). These images were registered to the NICFPS image using field stars to an accuracy of about 0 ". 05 , limited by optical distortions. The time-intervals between the first image and the second and third images are 6.33 and 12.19 years.

Proper motions were determined using difference-squared cross-correlation technique of Currie et al. (1996). All images were re-sampled to the pixel-scale of the 2004 epoch NICFPS image and blinked to identify moving features. Measurement boxes containing between 50 and 500 pixels were defined on the 2004 epoch image as shown in Figure 1. On the priorepoch images, a larger measurement box with 6 additional pixels beyond each boundary was defined at each location. Slowly-varying background emission was removed by fitting a twodimensional cubic function and subtraction of the result. The smaller box is shifted within the larger box for all possible $x$ and $y$ pixel-offsets and subtracted from the larger box. The square of the sum of all pixel-to-pixel differences is assigned to the pixel that corresponds to the $x$ and $y$ offset in an "offsets" image consisting of all possible pixel shifts. The location of the minimum in this two-dimensional array is an estimator of the $x$ and $y$ offset that results in the best match between the intensities in the boxes defined on the first and second epoch images. Proper motions are estimated using the centroid of a two dimensional gaussian fit to this "offsets" image. Field stars were used to estimate a mean proper motion error of about $15 \mathrm{~km} \mathrm{~s}^{-1}$ for bright compact sources for both the 2004 - 1999 and 2004 - 1992 comparison.

Most proper motion vectors point directly away from the OMC1 cloud core. However, about $10 \%$ of the vectors differ by more than $30^{\circ}$ from the expected direction of motion away from the point of origin of the outflow. The 4 boxes located in the lower-right portion of Figure 1 trace a highly collimated chain of $\mathrm{H}_{2}$ bow-shocks that emerge at position angle PA $\sim 320^{\circ}$ from the OMC1-S core located about 90" south OMC1. Another collimated outflow crosses the northwestern lobe of the OMC1 outflow at position angle PA $\sim 240^{\circ}$ to $250^{\circ}$. Its brightest southwest-facing bow shock is located at $\mathrm{J}(2000)=5^{h} 35^{m} 11 .{ }^{s} 6,-05^{\circ} 20^{\prime} 54^{\prime \prime}$. The orientation of this shock indicates that the source is likely to be located towards the 
northeast in the "Integral Shaped Filament" which contains the highest column density of molecular gas (Johnstone \& Bally 1999).

Figure 1 shows the $\mathrm{H}_{2}$ proper motion vectors. The fastest motions are located at the greatest distance from the OMC1 core while the slower motions are located closer in. Thus, to first order, the motions are consistent with velocity being proportional to distance (e.g. a "Hubble flow"), an indication of an explosion.

Figure 2 shows the proper motions of $2.122 \mu \mathrm{m} \mathrm{H}_{2}$ features measured by Cunningham (2006, and this work), $1.644 \mu \mathrm{m}$ [FeII] (Lee \& Burton 2000), and Herbig-Haro objects traced by $\mathrm{H} \alpha$, [OI], [NII], and/or [SII] (Doi, O’Dell, \& Hartigan 2002) as a function of distance from the point of origin of the three high-velocity stars. $\mathrm{H}_{2}$ tends to only trace features with relatively low proper motions below about $70 \mathrm{~km} \mathrm{~s}^{-1}$. Presumably this is consequence of $\mathrm{H}_{2}$ dissociation in magnetized C-shocks with speeds in excess of about $70 \mathrm{~km} \mathrm{~s}$. For non-magnetized J-shocks, $\mathrm{H}_{2}$ dissociates at shock speeds of $10-30 \mathrm{~km} \mathrm{~s}^{-1}$. The pair of $250 \mathrm{~km} \mathrm{~s}^{-1} \mathrm{H}_{2}$ features located about $0.1 \mathrm{pc}$ from the expansion center in Figure 2 may trace reverse shocks moving into dense molecular clumps that have high velocities. H $\alpha$ excitation requires shock speeds of at least $60 \mathrm{~km} \mathrm{~s}^{-1}$ while the $\lambda 5007 \mathrm{~A}$ line of [OIII] requires shocks with speed in excess of at least $150 \mathrm{~km} \mathrm{~s}^{-1}$. Most visual-wavelength forbidden transitions such as the $\lambda 6717 / 6731$ [SII] lines trace the cooling layers behind fast shocks. [FeII] and the visual-wavelength tracers are commonly associated with features having velocities up to $425 \mathrm{~km} \mathrm{~s}^{-1}$.

Figure 2 demonstrates that some proper-motion features have apparent ages between 500 and 1,000 years. Dynamical ages are determined from the distance traveled divided by the magnitude of the proper motion. Unlike age determination from radial velocities, knowledge of the inclination of the flow vector is not required. For example $\mathrm{HH} 210$, the fastest $\mathrm{HH}$ object associated with the $\mathrm{BN} / \mathrm{KL}$ outflow, has a dynamic age of 605 years assuming no deceleration. Given that most shocks are likely to have been decelerated by interactions with their environment, Figure 2 shows that the ages of many features in the $\mathrm{BN} / \mathrm{KL}$ outflow are consistent with ejection about 500 years ago, similar to the time elapsed since the ejection of the three high-velocity stars, BN, I, and n.

Figure 3 shows the apparent point of origin of the $\mathrm{H}_{2}$ proper motions in the OMC1 outflow. The left panel is made by backward tracing of the proper motion vectors, effectively marking their trajectories in the absence of deflections. The peak in the density of intersecting trajectories provides the best estimator for the point of origin of the measured motions. The "intensity" of each trail is weighted by the speed. Thus, large motions, which can be measured more precisely than small motions, have a greater weight. The image in the right panel has been boxcar-smoothed by 31 pixels $\left(25^{\prime \prime}\right)$ to aid visualization of the intersection 
point. The point of origin of the proper motions is located at $\mathrm{J}(2000)=5^{h} 35^{m} 14 .{ }^{s} 5,-5^{\circ} 22^{\prime}$ $23^{\prime \prime}$, with a $1 \sigma$ uncertainty of about $5^{\prime \prime}$. This location is offset by about $5^{\prime \prime}$ to the northeast of the apparent ejection center of the massive stars and $9^{\prime \prime}$ north of the current location of radio source I. Zapata et al. (2010) found an expansion center based on interferometric $\mathrm{CO} \mathrm{J}=2-1$ observations at $\mathrm{J}(2000)=5^{h} 35^{m} 14 .{ }^{s} 37,-5^{\circ} 22^{\prime} 27 .{ }^{\prime \prime} 9$, about $5^{\prime \prime}$ due south the expansion center derived from our proper motions, but consistent within the uncertainty estimates.

\section{Runaway stars, Dynamical Decay, and the Origin of Explosive Outflows}

High velocity runaway stars are relatively common among massive O and early B stars but rare among lower-mass stars (Gies \& Bolton 1986; Gies 1987). While approximately $30 \%$ of $\mathrm{O}$ stars are runaways with velocities greater than $30 \mathrm{~km} \mathrm{~s}^{-1}$ with respect to their birth clusters and associations, less than $2 \%$ of B and A stars have such velocities (Stone 1991; Schilbach \& Roser 2008; Zinnecker \& Yorke 2007). Many (possibly most) massive runaway stars were ejected by three-body encounters between a single star and a massive binary (Gvaramadze \& Gualandris 2010), four-body encounters between a pair of massive binaries (Poveda, Ruiz, \& Allen 1967), or the decay of non-hierarchical multiple star systems (van Albada 1968; Valtonen \& Mikkola 1991; Aarseth et al. 1994; Sterzik \& Durisen 1995, 1998). Such three- or four-body encounters are most likely to occur in very dense, young star clusters. The runaway stars AE Aur and $\mu$ Col, ejected with speeds of over $100 \mathrm{~km} \mathrm{~s}^{-1}$ about $2.5 \times 10^{6}$ years ago (Hoogerwerf, de Bruijne, \& de Zeeuw 2001) from the NGC 1980 cluster located about $30^{\prime}$ south of the Orion Nebula, provide a prototypical example. This event produced the massive colliding-wind X-ray binary ८ Ori (Gualandris, Portegies-Zwart, \& Eggleton 2004).

Non-hierarchical multiple star systems, triple or higher-order multiples in which the time-averaged distances between components are similar are dynamically unstable. Numerical experiments show that non-hierarchical systems tend to rearrange into hierarchical configurations consisting of a compact binary and either ejected stars or ones in elongated orbits with a much larger semi-major axis than the binary. Non-hierarchical systems tend to decay in about 100 crossing times; the crossing time is roughly given (in years) by $t_{c r} \sim 0.17\left(R^{3} / M\right)^{1 / 2}$ where $R$ is the characteristic length scale of the system in $\mathrm{AU}$ and $M$ is total system mass in Solar masses (Anasova 1986; Sterzik \& Durisen 1995). Many low-mass pre-main sequence stars are born in such non-hierarchical multiples (Reipurth 2000). Among massive stars, Trapezium-like groups tend to be non-hierarchical and subject to dynamical decay. 
Both the ejection of massive stars and the launch of the OMC1 BN/KL outflow may be the result of the dynamic interaction and rearrangement of a system of massive stars in OMC1 about 500 years ago. The kinetic energy originates from the release of gravitational potential energy accompanying the dynamic formation of a compact binary. According to the Virial theorem, one-half of the (negative) potential energy of the binary goes into orbital motion of its constituent stars. The other half is removed from the system, and is available to accelerate the binary, other stars involved in the interaction, and surrounding gas. The most massive component of the system, which is the slowest moving object, is likely to be the binary. The binding energy of a binary consisting of masses $m_{1}$ and $m_{2}$ with an orbital semi-major axis $a, E_{b}=-G m_{1} m_{2} / 2 a$, must be twice the (negative) sum of the observed kinetic energies in the outflow and ejected stars.

Assuming that source I consists of two $10 \mathrm{M}_{\odot}$ stars, to release $2 \times 10^{47}$ (or $6 \times 10^{47}$ ) ergs of free kinetic energy, its members must have a semi-major axis less than 2.2 (or 0.74 ) AU with an orbital period shorter than 2 (or 0.4 ) years. The perihelion velocity of the stars must be at least 33 (or 55) $\mathrm{km} \mathrm{s}^{-1}$ and the binding energy of the binary must be less than $-4 \times 10^{47}$ (or $-12 \times 10^{47}$ ) ergs. If either BN or $\mathrm{n}$ is the binary, the binary separation must be much smaller to provide the energy for the ejection of the three stars plus outflow. Stellar ejection removed a total of about 22 to $36 \mathrm{M}_{\odot}$ of stars and least $8 \mathrm{M}_{\odot}$ of gas. Thus, this event removed 30 to $50 \mathrm{M}_{\odot}$ from the OMC1 core. The point of origin of the ejected stars is located at the center of the BN/KL outflow about $4^{\prime \prime}$ ( $\sim 1700 \mathrm{AU}$ in projection) northwest of the present location of radio source $\mathrm{I}$.

Momentum conservation requires that the vector sum of the stellar and outflow momenta sum to zero in the rest frame of the parent cloud. Thus, the runaway single stars and the dynamically formed binary are most likely to be ejected from their star-forming clump. Removal of the stars implies that the envelope whose motion was dominated by the gravity of the stellar system before ejection can become unbound after ejection. Multi-body encounters tend to eject the outer portions pre-existing circumstellar disks at radii larger than about $1 / 3$ to $1 / 2$ of the periastron separation. Thus, the outer parts of disks around the stars that form the binary and the envelope surrounding the disrupted cluster will be ejected with roughly their respective orbit speeds.

Three distinct mechanisms may be involved in releasing gas from the gravitational potential well. First, the disruption and ejection of the outer-parts of circumstellar disks during the final dynamic encounter leading the binary formation. Second, recoil of the pre-existing circum-cluster envelope following stellar ejection. Finally, the release of stored magnetic stress. Each process is a consequence of the removal of gravitational attraction on a time-scale given by the relevant crossing time. Disk disruption occurs within a time-scale 
$t_{\text {cross }} \approx r / V<<1$ year at $r=1 \mathrm{AU}$ for $V \sim 100 \mathrm{~km} \mathrm{~s}^{-1}$, the Kepler speed around a $10 \mathrm{M}_{\odot}$ star. At $r=100 \mathrm{AU}$, stellar ejection with a mean speed of $V=20 \mathrm{~km} \mathrm{~s}^{-1}$ implies that the envelope gas would be unbound several decades. In both cases, the expulsion velocity would be comparable to the Kepler speed prior to ejection, or about $20 \mathrm{~km} \mathrm{~s}^{-1}$ for an envelope initially orbiting $40 \mathrm{M}_{\odot}$ of stars at $r=100 \mathrm{AU}$ to over $100 \mathrm{~km} \mathrm{~s}^{-1}$ for parts of disks ejected from within $1 \mathrm{AU}$ of a $10 \mathrm{M}_{\odot}$ star. These mechanisms are explored in more detail below.

\subsection{Formation of a Non-Hierarchical Massive Multiple in OMC1}

OMC1 spawned at least 20 to over $40 \mathrm{M}_{\odot}$ of stars. Assuming a typical star-formation efficiency of $25 \%$ to $50 \%$ typical of massive cluster forming clumps, the OMC1 proto-cluster clump must have initially contained at least $M_{\text {clump }} \sim 100 \mathrm{M}_{\odot}$ of gas. As a fiducial example, consider a clump with an 'isothermal' density distribution $\rho(r)=\rho_{0}\left(r / r_{0}\right)^{-2}$ for radius $\mathrm{r}$ less than the clump outer radius $R$. A clump with mass $M_{\text {clump }}=100 \mathrm{M}_{\odot}$ inside $R=$ $0.1 \mathrm{pc}$ implies mean orbital velocity (which is independent of $r$ ), $V \approx\left(G M_{\text {clump }} / R\right)^{1 / 2}=$ $\left(4 \pi G \rho_{0}\right)^{1 / 2}=2.1 \mathrm{~km} \mathrm{~s}^{-1}$ where $\rho_{0} \approx 5.0 \times 10^{16} \mathrm{~g} \mathrm{~cm}^{-3}$ would be the density at $r_{0}=1 \mathrm{~cm}$ if the power-law distribution were extended to this radius. In this example, the density at $r=5,000$ AU from the center is $n\left(H_{2}\right) \approx 2 \times 10^{6} \mathrm{~cm}^{-3}$ and the crossing-time is $t_{\text {cross }} \sim R / V<5 \times 10^{4}$ years. The most massive protostellar objects are likely to either form in the center where the density and pressure are the greatest, or if they form away from the center, to rapidly migrate to the center of the potential due to orbit decay (Zinnecker 1982; Bonnell et al. 2001).

Orbits can decay due to Bondi-Hoyle (BH) accretion as they move though clump gas and dynamic-friction caused by the gravitational pull of the wake produced by converging streamlines behind the YSO. The BH accretion rate is roughly $\dot{M}_{B H}=\pi G^{2} M_{Y S O}^{2} \rho(r) /\left(V_{*}^{2}+\right.$ $\left.C_{s}^{2}\right)^{3 / 2}$ where $C_{s}$ is the effective sound speed (which includes the effects of turbulent motion and magnetic support) in the gas (usually $<1 \mathrm{~km} \mathrm{~s}^{-1}$ ). A star moving with a speed $V=2.1$ $\mathrm{km} \mathrm{s}^{-1} 5,000 \mathrm{AU}$ from the center of the above gas distribution would accrete at a rate $\dot{M}_{B H} \approx 9 \times 10^{-7} \mathrm{M}_{\odot} \mathrm{yr}^{-1}$ for a $1 \mathrm{M}_{\odot}$ YSO and $\dot{M}_{B H} \approx 2 \times 10^{-5} \mathrm{M}_{\odot} \mathrm{yr}^{-1}$ for a $5 \mathrm{M}_{\odot}$ YSO. As illustrated by the simulation described in the next paragraph, the most massive objects grow most rapidly, doubling their mass and migrating to the center of the cloud within few $\times 10^{5}$ years. Fragmentation (McKee \& Tan 2002; 2003; Krumholz 2006) or the capture of sibling stars (Moeckel \& Bally 2006; 2007a,b) may produce multiple systems. These processes could have resulted in the assembly of a sub-cluster of massive stars and binaries in the center of the core.

Figure 4 shows a sample result of a simplified numerical model illustrating the combined 
effects of Bondi-Hoyle accretion onto protostellar seeds, orbit decay, formation of a nonhierarchical system of massive stars, and dynamical ejection. This simulation starts with a $50 \mathrm{M}_{\odot}$ clump of gas in a Plummer potential given by $\Phi(r)=-G M / \sqrt{\left(r^{2}+a^{2}\right)}$ where $M$ is the total mass and $a=0.025 \mathrm{pc}$ is the core radius. (This potential is used to avoid the singularity at the center of an isothermal sphere). The clump is non-rotating with an internal velocity dispersion (effective sound speed) given by the local Virial velocity so that, to first order, the clump is stable to global gravitational collapse. At the start of the simulation, 20 protostellar seeds, each having an initial mass of $0.5 \mathrm{M}_{\odot}$, are distributed randomly in the clump. Their velocity distribution is virialized in the combined gravitational potential of the stars and gas and their velocity vector orientations are random. The simulation was run 100 times, each with a different randomly chosen set of initial locations and velocities for the seeds.

Stellar motions are calculated from the gravitational forces exerted by other stars and the static gas cloud, modified by the effects of $\mathrm{BH}$ accretion. The inter-stellar forces are directly calculated, and the orbits are integrated with a global but variable time-step using a $(7,8)$ order Runge-Kutta pair (Prince and Dormand 1981). The code is a standard nbody integrator with the addition of a static potential, identified with the natal gas, that can be accreted onto protostellar seeds. The code follows the stellar mass growth and orbit evolution in the combined gravitational potential of the clump plus embedded stars. Similar approaches have been used by Moeckel and Clarke (2010) and Baumgardt and Klessen (2010). The protostellar seeds experience $\mathrm{BH}$ accretion from the clump and grow in mass with the accretion radius set to the minimum of the $\mathrm{BH}$ radius or one-third the distance to the closest neighboring star. To keep the computations simple, the response of the gas to the passage of the stars is not modeled. This simplification is reasonable because the zone influenced by the passage of a protostellar seed is restricted to its gravitational radius, $r_{G}=G_{*} M / V_{*}^{2}$ $\left(\sim 200 \mathrm{AU}\right.$ for $M_{*}=1 \mathrm{M}_{\odot}$ and $\left.V_{*}=2 \mathrm{~km} \mathrm{~s}^{-1}\right)$. Random motions in the clump will tend to fill-in the cavity formed by the passage of a star on a time scale $\tau \sim r_{G} / C_{s} \geq 10^{3}$ years but much less than the $10^{5}$ year evolution time-scale of the protostars and clump. Thus, it is assumed that on average, the envelope remains spherical and smooth. However, as gas is accreted onto the stars the mass of the gas cloud, and thus the contribution of the gas to the gravitational potential, is reduced accordingly.

Protostars located in the densest portion of the clump and/or those that by chance have the lowest relative velocity with respect to the gas tend to grow in mass most rapidly. In this simulation, accreted gas lowers the orbital angular momentum of the stars about the clump center since the clump is assumed to be non rotating. Thus, as they grow in mass, their orbits decay and the most massive objects migrate to the clump center. The most rapidly growing protostars form a non-hierarchical system near the center. As they 
come to dominate the mass in this region, mutual gravitational interactions reconfigure the system into a hierarchy consisting of one or more tightly bound compact binaries plus ejected high-velocity stars. Multiple runs of the simulation with different randomly chosen initial locations and velocities almost always produce mass-seggregated, non-hierarchical systems of massive stars at their centers. This model illustrates one plausible scenario for the formation of a non-hierarchical system of massive stars and their subsequent ejection. Three essential features are missing from this simplified model; the dynamic ejection of gas from the central region by the orbital motion of the massive stars, the presence of accretion disks, and the streamers that transport mass from the inner-boundary of the envelope onto these disks. Previous simulations of binary star formation have shown these features to be present (e.g. Lubow \& Artymowicz 1996).

In a multiple star system, matter that enters the region where the stars orbit each other tends to be expelled by gravitational torques. As a result, gas bound to the system is likely to be organized into two components; an extended outer envelope having an inner boundary with a radius somewhat larger than the semi-major axis of the largest stellar orbit, and inner disks surrounding the stars with outer radii several times smaller than the periastron separations (Artymowicz \& Lubow 1994; Günther \& Kley 2002). For pre-decay interstellar separations of $100 \mathrm{AU}$, matter located within $\sim 300 \mathrm{AU}$ of the cluster would either be accreted onto disks with outer radii less than about $30 \mathrm{AU}$ or be expelled to beyond 300 AU. Gas in an envelope bound to a $40 \mathrm{M}_{\odot}$ cluster of stars with an inner radius of $300 \mathrm{AU}$ would have Kepler speeds less than $11 \mathrm{~km} \mathrm{~s}^{-1}$. The outer boundary where the cluster has significant influence on the envelope can be defined by the radius where the gravitational influence of the cluster falls below the velocity dispersion, about 2 to $3 \mathrm{~km} \mathrm{~s}^{-1}$ for OMC1. Disks with 30 AU outer radii orbiting $10 \mathrm{M}_{\odot}$ stars would have Kepler speeds greater than 17 $\mathrm{km} \mathrm{s}^{-1}$. Since non-hierarchical multiples likely have chaotic orbits, disks may be truncated at smaller radii. Gas falling from the outer envelope onto individual circumstellar disks may form transient streams in the region of avoidance.

Figures 5 illustrates a possible scenario for the evolution of the OMC1 cloud core. Figure 5a shows the formation of several protostellar seeds destined to become massive stars as they continue to accrete from the clump and sink to its center to form a non-hierarchical multiple system. Figure 5b shows the multiple system after it has cleared the orbit zone. Tidal streams can continue to feed the growth of compact circumstellar disks with outer radii smaller than the typical periastron separation. Figure $5 \mathrm{c}$ shows the aftermath of the dynamic interaction that ejected the stars from the clump. 


\subsection{Recoil of the Envelope; Slow Ejecta}

Proper motions (Gomez et al. 2005, 2008) indicate that the stars BN, I, and n originated from a region less than $400 \mathrm{AU}$ in diameter 500 years ago. To evaluate the kinetic energy of the envelope before stellar ejection, consider a sphere of gas with a central hole containing a cluster with mass $M_{c l}=20$ to $40 \mathrm{M}_{\odot}$ ('cl' stands for cluster). Assume that the envelope density is given by $\rho(r)=\rho_{0} r^{-\alpha}$ between an inner boundary $r_{i n}$ and an outer boundary $r_{\text {out }}$. The initial total mass of the envelope may have been around $M_{e}=100 \mathrm{M}_{\odot}$. As it formed stars, the envelope mass would decrease to $M_{e}-M_{c l} \approx 60$ to $80 \mathrm{M}_{\odot}$. Appendix 1 presents an analytic formula for estimating the kinetic energy stored in the envelope due to orbital motion about the central cluster.

Assuming an envelope velocity dispersion of $\sigma=2 \mathrm{~km} \mathrm{~s}^{-1}$, the gravitational potential of the stars dominated motions located within $r_{G}=G M_{c l} / \sigma^{2} \approx 4,400$ to $9,000 \mathrm{AU}$ (11" to $21^{\prime \prime}$ at the $414 \mathrm{pc}$ distance to OMC1) for 20 and $40 \mathrm{M}_{\odot}$ of stars, respectively. Clumps within the envelope at a distance $r$ from the center would move with velocities of order $V(r)=\left(G\left[M_{c l}+M_{g a s}(<r)\right] / r\right)^{1 / 2}=3$ to about $20 \mathrm{~km} \mathrm{~s}^{-1}$ in the potential. For a $0.1 \mathrm{pc}$ outer radius sphere containing $60 \mathrm{M}_{\odot}$ of gas and $40 \mathrm{M}_{\odot}$ of stars, the mass of gas interior to $5,000 \mathrm{AU}$ is about $7 \mathrm{M}_{\odot}$ if the power-law index of the density distribution is $\alpha=1.5$, and almost $14 \mathrm{M}_{\odot}$ if $\alpha=2$. Thus the total mass in this region is comparable to the low velocity portion of the BN/KL outflow.

Following stellar ejection, the inner envelope where the stellar mass dominated the potential will be moving faster than the escape speed from the remaining mass. Thus, this mass will expand. Assuming that each mass element moves with the Kepler speed corresponding to its orbit radius before ejection, the average kinetic energy at radius $r$ in an infinitesimal radial increment $d r$ is $d \epsilon=0.5 V_{K}^{2}(r) d M$ where $d M=4 \pi r^{2} \rho(r) d r$ and $V_{K}^{2}(r)=G\left[M_{c l}+M_{e}(r)\right] / r$, where $M_{c l}$ is the mass of the ejected stars and $M_{e}(r)$ is the mass of envelope gas inside radius $r$. This can be integrated over radius to determine the total kinetic energy in orbital motion. Following stellar ejection, as this gas expands, it had to climb out of its own potential. The free kinetic energy of the expanding envelope is given by the orbital kinetic energy before stellar ejection minus the energy required to climb out of its own potential well after stellar ejection.

Appendix 1 gives an expression for the free kinetic energy of a recoiling envelope which, prior to stellar ejection, had mass $M_{e}$ with a power-law radial density profile between an outer radius $r_{\text {out }}$ and inner radius $r_{\text {in }}$ bound to a central cluster with mass $M_{c l}$. Following stellar ejection, the envelope has more kinetic energy than (negative) gravitational potential due to its own mass. The orbital kinetic energy due to the central cluster is represented by the first term in equation A1-1. The self-energy of the envelope is represented by the 
second term in equation A1-1. Following dynamic ejection, the orbital motion of clumps in the envelope exceed the self-binding energy. Equation A1-2 gives the final kinetic energy of the envelope after ejection of the central cluster. Table 1 lists these kinetic energies $\left(10^{45}\right.$ to $10^{46} \mathrm{ergs}$ ) for parameters considered plausible for OMC1.

For steeper density gradients, a larger fraction of the envelope is concentrated deeper in the potential well of the stars. The kinetic energy of the ejected envelope can be increased by migrating mass closer to the center of mass prior to stellar ejection. For example, if the entire mass of an inner envelope with $\mathrm{M}_{e}=10 \mathrm{M}_{\odot}$ were placed in an orbiting ring with a radius $r$, its orbital kinetic energy would be $G M_{c l} M_{e} / 2 r \approx 3.5 \times 10^{46}$ ergs for $r=100 \mathrm{AU}$, $M_{c l}=40 \mathrm{M}_{\odot}$. The orbital momentum of such a ring, $P_{e}=M_{e} V_{K}\left(r_{i n}\right)$ would be about $190 \mathrm{M}_{\odot} \mathrm{km} \mathrm{s}^{-1}$, comparable to the outward directed momentum in the BNKL outflow. The gravitational self energy of the inner envelope is of order $G M_{e}^{2} / r \approx 1.8 \times 10^{46}$ ergs (the exact number depends on the density and dimensions of the ring). Thus, in this example, the outflowing kinetic energy of the envelope after stellar ejection is about one half of the

orbital kinetic energy. The fraction of the orbital energy available as free kinetic energy in an outflow increases as the ratio of the envelope mass to the ejected cluster mass decreases.

\subsection{Disruption of Disks; Fast Ejecta}

The destruction and ejection of circumstellar matter during the final penetrating encounter leading to the decay of a massive multiple star system that ejects several members can release an order-of-magnitude more gravitational potential energy than the gentle recoil of the surrounding weakly bound envelope. During the final stellar encounter that formed an AU-scale binary whose gravitational potential energy expelled the stars from the region, their circumstellar disks would likely be destroyed and ejected. If the binary consists of a pair of $10 \mathrm{M}_{\odot}$ stars separated by 0.7 to $2.2 \mathrm{AU}$ as required by the energetics of the OMC1 ejection, the outer radius of any surviving disk around either star can be no greater than about 0.1 to $0.5 \mathrm{AU}$. Matter ejected from this region in orbit around a $10 \mathrm{M}_{\odot}$ star would have a velocity of order 100 to $300 \mathrm{~km} \mathrm{~s}^{-1}$. In an encounter between two or three massive stars, gravity will accelerate the stars to about this speed if the periastron is about an AU. In a prograde encounter between a star and matter in another star's disk, the head-on flow can "slingshot" this material to about twice the relative encounter speed. Moeckel and Bally (2006, 2007a, b) presented SPH simulations of the gravitational and hydrodynamic interaction of single stars crashing through the disk of a massive star on hyperbolic orbits. They found that for a wide range of parameters, the incoming stars could be captured into an eccentric, non-coplanar, elliptical orbit. The interaction truncates the disk, and ejects its 
outer parts.

The fastest observed ejecta in the BN/KL outflow have speeds between 200 to 400 $\mathrm{km} \mathrm{s}^{-1}$ but the total mass in the fast components above $100 \mathrm{~km} \mathrm{~s}^{-1}$ is less than $0.1 \mathrm{M}_{\odot}$. We propose that this ejecta was produced by the disruption of circumstellar disks and launched from radii $r_{d}<1 \mathrm{AU}$ from around $\sim 10 \mathrm{M}_{\odot}$ stars. Assume that individual circumstellar disks can be characterized by a power-law surface density of the form $\Sigma_{d}=\Sigma_{0} r^{-\beta}$ where the subscript $d$ signifies the inner disk (as opposed to the extended envelopes). Appendix 2 gives an expression for the free kinetic energy of high-velocity 'shrapnel' created by the disruption of a circumstellar disk during the final encounter that led to the formation of a compact binary. Table 2 lists the kinetic energies of this debris as a function of plausible pre-ejection disk parameters. For power-law disks with $\beta$ ranging from 0.5 to 1.5 , outer radii $r_{d_{\text {_out }}}=10 \mathrm{AU}$, inner radii ranging from $r_{d_{-} \text {in }}=0.05$ to $0.5 \mathrm{AU}$, total disk masses of 0.5 to $1.0 \mathrm{M}_{\odot}$ orbiting a $M_{*}=10 \mathrm{M}_{\odot}$ star, the kinetic energies of ejecta after stellar ejection range from $10^{46}$ to $10^{47} \mathrm{ergs}$, an order of magnitude greater than the energies in the recoiling envelope. Steeper surface density profiles concentrate matter toward the star and increase the kinetic energy per unit mass stored in the disk. Table 2 lists the energies of ejected disk gas. If the circumstellar material surrounding several stars are ejected in this manner, the kinetic energy and momentum requirements of the BNKL outflow can be met by the disruption of such disks alone. The disruption and ejection of the inner disks during the final encounter that ejected OMC1's massive stars could have powered the fastest ejecta in the BN/KL outflow.

\subsection{The Contribution of Stored Magnetic Stress}

The birth and subsequent orbital motion of a non-hierarchical cluster of 4 or 5 massive stars in OMC1 over $10^{5}$ or $10^{6}$ years may have led to the amplification of ambient magnetic fields in both the envelope surrounding the cluster and in individual circumstellar disks. Fields can grow until either magnetic-reconnection or resistive dissipation in the charged component of the weakly ionized plasma come to equilibrium with the dynamo (LyndenBell 1996; Wheeler, Meier, \& Wilson 2002; Vasil \& Brummell 2008, 2009). The orbital motion of the protostars in the cluster gravitational potential and the orbital motion of gas in individual circumstellar disks may each contribute to the amplification of the magnetic fields (Käpylä et al. 2008).

When equipartition is reached, $B^{2} \approx 8 \pi \rho(r) c_{s}^{2}$, where $\rho(r)$ is the local gas density and $c_{s}$ is the effective sound speed including turbulent motions. For the envelope having the powerlaw density profile described in Appendix 1, the equipartition magnetic energy density at 
radius $r$ is given by $\epsilon_{B e}=B^{2}(r) / 8 \pi=\rho(r) c_{s}^{2}$ where $c_{s}$ is the local effective sound speed (including thermal and turbulent motion) and the subscript $e$ refers to the envelope. Under this assumption, the magnetic energy in a shell at radius $r$ and thickness $d r$ is

$$
d E_{B e}=4 \pi r^{2} \epsilon_{B e} d r=r^{2} B_{e}^{2}(r) d r / 2=\rho_{e}(r) c_{s}^{2} d r=4 \pi \rho_{0} c_{s}^{2} r^{2-\alpha} d r
$$

which can be integrated from the inner edge of the envelope to a radius $r$ to give

$$
E_{B e}=\int_{r_{i n}}^{r} d E_{B e}=\frac{4 \pi \rho_{0} c_{s}^{2}}{3-\alpha}\left(r^{3-\alpha}-r_{i n}^{3-\alpha}\right) .
$$

Setting $r=r_{\text {out }}$ gives $E_{B e}=M_{e} c_{s}^{2}$. For $M_{e}=60 \mathrm{M}_{\odot}$ and $c_{s}=2 \mathrm{~km} \mathrm{~s}^{-1}$ this results in $E_{B e} \approx 5 \times 10^{45} \mathrm{ergs}$ for the equipartition magnetic energy that could be stored in the envelope.

If magnetic stress reaches equipartition with the effective sound speed within circumstellar disks, a similar calculation yields $E_{B d}=M_{d} c_{d}^{2}$ where the subscript $d$ refers to the disk quantities. The effective sound speeds in the disks are likely to be considerably larger due to proximity to the massive stars. Using $c_{s}=10 \mathrm{~km} \mathrm{~s}^{-1}$ gives $E_{B d}=2.0 \times 10^{45} M_{1} c_{10}^{2}$ ergs for the equipartition magnetic energy that could be stored in a disk. Here $M_{1}$ is in units of $1 \mathrm{M}_{\odot}$ and $c_{10}$ is in units of $10 \mathrm{~km} \mathrm{~s}^{-1}$.

Magnetic fields may become stronger than equipartition with the local pressure in the disk or envelope. Keplerian shear can wind-up the field so that its energy approaches the gravitational potential, a circumstance discussed in the context of circumstellar disks by Shu et al. (2008). The condition that Alfven speed (or effective sound speed) is equal to the gravitational escape speed sets an upper bound on the field-strength. Thus, $V_{A}^{2}=$ $B^{2} / 4 \pi \rho \approx G M / r$ where $M$ is the mass enclosed inside radius $r$. In this case, the field could have stored more than $10^{47}$ ergs of magnetic energy in the volume inhabited by the cluster. The removal of about $40 \mathrm{M}_{\odot}$ of stars would have left the circum-cluster gas severely magnetically over-pressured. The release of magnetic energy, in effect a 'magnetic bomb' (Matt, Frank, \& Blackman 2004, 2006), may have been a major source of energy for launching the OMC1 outflow. The strongest fields are likely to be generated by shear dynamos in the innermost parts of circumstellar disks surrounding individual massive stars. Future Zeeman measurements of clumps containing sub-clusters of massive protostars using the 24 to $26 \mu \mathrm{m}$ iron lines with SOFIA may determine if energetically significant, gauss-strength fields can be generated in such environments (R. Crutcher - private communication). 


\section{Discussion}

The BN/KL outflow is velocity segregated with relatively slow gas confined to a few tens of arc-seconds from radio source I and the fastest ejecta, located near the tips of the [FeII] and $\mathrm{H}_{2}$ fingers about $120^{\prime \prime}$ northwest and southeast of the core region. Proper motion measurements of visual-wavelength HH objects (such as HH 205 through 210), near-infrared emission from shock-excited [FeII] and $\mathrm{H}_{2}$ (Figures 1 and 2), and the interferometric $\mathrm{CO}$ $\mathrm{J}=2-1$ study of Zapata et al. (2010) indicate that the BN/KL outflow was generated by an explosive event about 500 years ago. This age coincides with the dynamical re-arrangement of the massive stars in OMC1 during which radio sources I, n, and BN were ejected (Gomez et al. 2005; 2008). The slowest star, radio source I, is probably a compact binary consisting of roughly $10 \mathrm{M}_{\odot}$ stars with a separation less than about 2 AU. Future long-baseline radio interferometry, or precision radial velocity measurements in the infrared may determine if this conjecture is true. It is proposed that the formation of this binary and the consequent release of 2 to $6 \times 10^{47}$ ergs of gravitational potential energy powered both the motion of the stars and the supersonic expulsion of gas in the BN/KL outflow. The slowest ejecta may have been produced at large radii as the pre-ejection orbits of clumps in the envelope became unbound following stellar ejection. The fastest ejecta may have originated from near the center of the potential well vacated by the stars. This situation is similar to that postulated by McCaughrean and Mac Low (1997) and Stone et al. (1995), where the $\mathrm{H}_{2}$ fingers are generated by the interaction between a slow, outer wind and a fast, later ejected flow. The estimates based on simple models of envelopes and circumstellar disks with power-law density distributions indicate that the gravitational energy stored in orbital motion prior to ejection can explain the energetics of the outflow following stellar ejection. However, numerical modeling of realistic configurations is need to determine of this hypothesis is valid.

The explosive OMC1 outflow consists of multiple fingers and wakes of $\mathrm{H}_{2}$ emission produced by the fastest ejecta plowing through the $\mathrm{OMC} 1$ core. The volume filling factor of the cavities created by this ejecta is likely to be lower than the enclosed volume, especially towards the southeast. Observations show that a reservoir of dense gas is located in the 'hot core' $5^{\prime \prime}$ to $20^{\prime \prime}$ southeast of the site of stellar ejection. Zapata et al. (2010) propose that the 'hot-core' may be blocking portions of the outflow in this direction. The bulk of the $\mathrm{H}_{2}$ emission is located to the northwest, consistent with more of the original core being blownout in this direction. The ejected stars moving south likely plow through the remaining dense material. 


\subsection{Post-Ejection Disks and Outflow Orientations}

Source I and possibly BN and n, are surrounded by circumstellar disks (Rodriguez, Zapata, \& Ho 2008). A polarized, infrared bipolar reflection nebula can be traced over $20^{\prime \prime}$ from BN. Simpson et al. (2006) argue that the polarization is produced by dichroic absorption by magnetically aligned dust in a foreground dust lane. Jiang et al. (2005) presented-adaptive optics-assisted polarimetric near-infrared images of the BN object and found a polarization pattern indicating illumination from BN. The nebula is bipolar with a symmetry axis oriented at $\mathrm{PA} \approx 36^{\circ}$ and a dark band parallel to BN's proper motion. The dark lane may be a "disk-shadow" (e.g. Pontoppidan \& Dullemond 2005) produced by opaque material close to the central star; thus the silhouette only provides an upper-bound on the disk outer radius. Such a disk must be oriented close to edge-on with an axis nearly orthogonal to BN's proper motion.

Radio source I is also surrounded by a nearly edge-on disk with an axis orthogonal to its apparent proper motion. The elongated $7 \mathrm{~mm}$ continuum emission associated with source I has been interpreted as a nearly edge-on disk rendered visible by collisional ionization and $\mathrm{H}^{-}$free-free opacity (Greenhill et al. 1998; Reid et al. 2007). The disk has a radius of about $50 \mathrm{AU}$ and an axis oriented towards $\mathrm{PA} \approx 45^{\circ}$. The thermal $\mathrm{SiO}$ emission at $86 \mathrm{GHz}$ (Plambeck et al. 2009) and $22 \mathrm{GHz} \mathrm{H}_{2} \mathrm{O}$ masers on scales of a few thousand AU indicate the presence of a very compact bipolar outflow with an age of only a few hundred years blowing towards the northeast and southwest along the suspected disk axis (Wright et al. 1995; Matthews et al. 2008; Beuther \& Nissen 2008; Plambeck et al. 2009).

Although source $\mathrm{n}$ is suspected to be surrounded by a disk, its orientation remains uncertain. Elongation in mid-infrared images suggest an axis towards the north-northeast, close to the axis of the elongation or double structure seen at radio wavelengths (Shuping et al. (2004).

Acceleration of the stars during the interaction that ejected them would have stripped away the outer parts of disks where the Kepler speed in less than than the stellar velocity. Thus, for BN and n, surviving disks would be truncated beyond about $10 \mathrm{AU}$. The final penetrating encounter during the decay of the non-hierarchical multiple star system may place even more stringent constraints on disk outer radii by ejecting material lying outside a radius of around $1 / 2$ to $1 / 3$ the periastron distance of the encounter. If source I consists of a pair of $10 \mathrm{M}_{\odot}$ stars, material beyond about an AU would be ejected. Some of this material may have remained bound to source I and fallen back to form a circum-binary disk. Such a disk would be confined to an annular region with a radius greater than 3 to $10 \mathrm{AU}$ set by the binary orbits, and less than about $50 \mathrm{AU}$ by the ejection velocity. The $7 \mathrm{~mm}$ continuum and $\mathrm{SiO}$ maser emission from source I indicates that it is currently surrounded by a disk with an 
outer radius of about $50 \mathrm{AU}$ with a northeast-southwest axis. A very young (dynamic age of a few hundred years) outflow emerges along this axis for a few arc-seconds. Did the source I disk survive dynamic ejection, or was it accumulated from the surrounding core within the last 500 years? These questions are considered in the following sub-section.

\subsection{Disks Accreted from the Surrounding Medium?}

In the proposed scenario, the currently observed disks either trace (i) material that has expanded from within a few AU of each star, (ii) disk debris ejected at less than escape speed that fell-back to re-form a new disk in the last 500 years, or (iii) was captured by Bondi-Hoyle accretion as the ejected stars plowed through remaining dense gas such as the 'hot core' in OMC1.

A star with mass $M_{*}$ moving with velocity $V$ through a cloud can accrete material lying within a cylinder defined by the gravitational radius $r_{G}=G M_{*} / V^{2}$ which is measured orthogonal to the stellar trajectory. After traversing a distance $L$ the amount of mass captured is about

$$
M_{B H}=\pi r_{G}^{2} L \rho=\pi \mu m_{H} n\left(H_{2}\right) L G^{2} M_{*}^{2} / V^{4}=2 \times 10^{-5} n_{5} L_{3} M_{10}^{2} V_{10}^{-4}
$$

where $\mu$ is the mean molecular weight, $m_{H}$ is the mass of hydrogen, $n_{5}$ is the number density of $\mathrm{H}_{2}$ in units of $10^{5} \mathrm{~cm}^{-3}, L_{3}$ is the path traversed by the star in units of $10^{3} \mathrm{AU}, M_{10}$ is the mass of the star in units of $10 \mathrm{M}_{\odot}$, and $V_{10}$ is the velocity of the star through the medium in units of $10 \mathrm{~km} \mathrm{~s}^{-1}$. Throop and Bally (2008) explored some consequences of $\mathrm{BH}$ accretion onto low-mass protostars as they moved through dense clouds. Moeckel and Throop (2009) modeled this late-phase BH accretion from a uniform density cloud onto a young star surrounded by an accretion disk using a hydrodynamic code with an isothermal equation of state but did not consider velocity or density gradients.

Bondi-Hoyle accretion tends to be subject to a "flip-flop" instability that leads to highly variable accretion rates and fluctuating orientations of the angular momentum vector of the flow (Shima et al. 1998; Fryxell \& Taam 1988; Ruffert 1987, 1999; Krumholz, McKee, \& Klein 2006). However, gradients in either velocity or density orthogonal to the stellar velocity give the flow vorticity with respect to the accretor. The resulting angular momentum can curtail accretion directly onto the moving mass because the flow tends to form a disklike structure. Ruffert (1997) presented 3D simulations of Bondi-Hoyle accretion from a medium having a velocity gradient while Ruffert (1999) considered flows from media with density gradients. Krumholz, McKee, \& Klein (2005) modeled accretion from a medium having vorticity. These studies show that Bondi-Hoyle accretion from a medium with a 
small velocity or density gradient is still subject to the "flip-flop" instability. However, large gradients tend to produce accretion disks with a relatively stable angular momentum vector orthogonal to the star's velocity (Ruffert 1999).

The BN object is moving away from the hot-core towards the northwest and has traversed about 4,000 AU since ejection. If the average density of the medium through which it moved had $n\left(H_{2}\right) \sim 10^{5} \mathrm{~cm}^{-3}$, a $30 \mathrm{~km} \mathrm{~s}^{-1}$ velocity implies that it could have swept-up about $M_{B H}=1.4 \times 10^{-6} \mathrm{M}_{\odot}$. If source $\mathrm{n}$ has a mass of $5 \mathrm{M}_{\odot}$, traversed 2,000 AU through a medium with a density of $10^{5} \mathrm{~cm}^{-3}$ with a velocity of $30 \mathrm{~km} \mathrm{~s}^{-1}$, it could have Bondi-Hoyle accreted $M_{B H}=1.2 \times 10^{-7} \mathrm{M}_{\odot}$.

Radio source I is moving towards the dense 'hot core'. Assuming that it has a mass 20 $\mathrm{M}_{\odot}$, is moving through a medium with $n\left(H_{2}\right)=10^{6} \mathrm{~cm}^{-3}$ with a velocity of $10 \mathrm{~km} \mathrm{~s}^{-1}$, and

has traversed a distance or order 1,000 AU, it could have swept-up as much as $M_{B H}=8 \times 10^{-4}$ $\mathrm{M}_{\odot}$. For source $\mathrm{I}$, assuming a mass $M_{I}=20 \mathrm{M}_{\odot}$, the time to cross the gravitational radius, $G M_{I} / V_{I}^{2} \sim 100 \mathrm{AU}$ at velocity $V_{I}=13 \mathrm{~km} \mathrm{~s}^{-1}$ is less than 40 years. The Kepler orbit time at a distance of $50 \mathrm{AU}$ from a $20 \mathrm{M}_{\odot}$ compact binary is about 80 years. Thus, any material accreted within the last several hundred years would have orbited at least three times at 50 AU. Thus, enough time has elapsed for accreted matter to have damped much of its motion orthogonal to its plane of symmetry and would resemble a disk.

Both BN and radio source I are moving close to the plane of the sky. If the accreted disk's angular momentum vector lies in a plane orthogonal to the stellar velocity vector, the apparent major axis of such a disk as projected onto the plane of the sky will be aligned with the proper motion vector. Observations show that the major axes of both the $7 \mathrm{~mm}$ continuum emission (Reid et. al. 2007) and the SiO maser emission (Goddi et al. 2009) from radio source I (which are interpreted as a nearly edge-on disk), and the putative disk associated with BN (Jiang et al. 2005) are parallel to their proper motions. These disk orientations are consistent with Bondi-Hoyle accretion onto the ejected stars from a medium having a significant density or velocity gradient orthogonal to the stellar motion.

\subsection{Is the Orion $\mathrm{BN} / \mathrm{KL}$ Outflow Unique?}

If such a mechanism operates in other hot cores and massive star forming regions, then it is expected that observations will reveal other outflow systems similar to Orion BN/KL. Such eruptive or explosive outflows will be associated with ejected, high-velocity massive stars whose time since ejection will be comparable to the outflow ages. The Spitzer Space Telescope detected $4.5 \mu \mathrm{m}$ emission from a wide-angle outflow having a morphology similar 
to Orion emerging from a high-luminosity $\left(\sim 10^{6} \mathrm{~L}_{\odot}\right)$ hot-core in G34.25+0.16 in the inner Galaxy (Cyganowski et al. 2008). Unfortunately, this flow and its source cloud core are highly obscured because it is located at a distance of about $5 \mathrm{kpc}$ in the Galactic Molecular Ring. Another possible example of an outflow having a morphology suggestive of an explosive origin is located in the NGC 7129 star forming region in Cepheus. This flow appears to originate from a moderate luminosity $\left(<10^{3} \mathrm{~L}_{\odot}\right)$ protostar. Source $\mathrm{G}$ in $\mathrm{W} 49$, which is the most luminous water maser outflow in the Milky Way, may be yet another example (Smith et al. 2009). Finally Sahai et al (2008) found evidence for interstellar bullets having a similar structure to the OMC1 fingers in the outflow from the massive young protostar IRAS 05506+2414.

\section{Conclusions}

The main conclusions of this study are:

1) New proper motion measurements show that some of the fastest and most distant $\mathrm{H}_{2}$ knots in the OMC1 BN/KL outflow have dynamical ages of 500 years, similar to the 500 year interval since dynamical decay ejected radio sources I, BN, and $n$ from a region located only a few arc-seconds from the center of the outflow. Thus, it is likely that the outflow was produced by the same event than ejected the massive stars BN, I, and $n$ form the core.

2) To within an order of magnitude, the kinetic energies of the outflow and ejected stars are comparable.

3) A scenario is explored in which the explosive OMC1 outflow is produced by the dynamical ejection of massive stars. Within the last few hundred thousand years, a compact non-hierarchical multiple system of massive stars or a pair of massive binaries must have formed in the OMC1 cloud core. A highly idealized numerical model is presented in which protostellar seeds grow by Bondi-Hoyle accretion from the parent clump. Seeds which by chance wander into the densest part of the core or have the lowest relative velocity with respect to the surrounding gas experience the highest accretion rates. They become massive, experience orbit decay, and migrate to the center of the model where they form a dynamically unstable multiple system.

4) The kinetic energy of the stars and the outflow could have been generated by release of gravitational binding energy accompanying the formation of a compact binary, most likely radio source I. If source I consists of a pair of $10 \mathrm{M}_{\odot}$ stars, then to produce the observed kinetic energy of the ejected stars and the outflow, between 2 to $6 \times 10^{46} \mathrm{ergs}$, the mean separation of the binary must be between 2.2 and $0.7 \mathrm{AU}$. 
5) In the proposed scenario, the outflow is driven by the energy and momentum stored in orbital motion prior to dynamical decay. Previous studies of gas flows in multiple-star systems show that orbiting material tends to be organized in a hierarchy consisting of tightly bound circumstellar disks with outer radii less than about $1 / 3$ of the periastron separation and relatively loosely bound envelopes with inner radii several times the apastron separation. Such a scenario is envisaged to to have been present in OMC1 prior to the ejection of its massive stars.

6) Stellar ejection may contribute to the formation of an explosive outflow by three mechanisms: Recoil of the envelope: The removal of stellar mass from the center of the envelope would result in the conversion of its orbital motion into linear motion. Disruption and ejection of circumstellar disks: The final penetrating encounter that formed a compact binary would eject the inner parts of pre-existing disks to produce the fastest ejecta. Release of magnetic stress: Magnetic energy potentially produced by shear-dynamo action in both circumstellar disks and the envelope might boost the velocities of the ejecta. In this scenario, the fastest ejecta is launched first from deep inside the gravitational potential of the decaying cluster. This material must plow through the slower-moving and later ejected envelope. Such interactions are prone to Rayleigh-Taylor type instabilities as shown by models of fast-winds slamming into slower, previously ejected winds. Order-of-magnitude energy estimates show that all three mechanisms are plausible, and that all three may contribute to the energy budget.

7) The ejected high-velocity stars may have accreted new circumstellar material as they traversed the dense OMC1 core. In the presence of gradients in the medium, the angular momentum vectors of accreting gas tend to be orthogonal to the stellar velocity vectors. Therefore, for stellar motions close to the plane of the sky, disk major axes will be aligned (in projection) with the stellar proper motion vectors, as observed for both radio source I and BN. Consequently, any recent outflow activity produced by the ejected stars will have axes orthogonal to the stellar proper motion vector. The youngest ejecta from radio source I as traced by $\mathrm{H}_{2} \mathrm{O}$ and $\mathrm{SiO}$ masers and $\mathrm{CO}$ has an axis orthogonal to its proper motion vector. The axis of symmetry of the near-infrared bipolar reflection nebula associated with $\mathrm{BN}$ is also orthogonal to its proper motion.

Future numerical hydrodynamic or magneto-hydrodynamic modeling of a massive-star forming clump in which a non-hierarchical system experiences a dynamical decay are needed to determine if the proposed scenario can indeed result in the generation of a powerful, explosive outflow such as is observed in Orion.

This work was supported by NSF grant AST0407356 and the CU Center for Astrobiology 
funded by NASA under Cooperative Agreement no. NNA04CC11A issued by the Office of Space Science. This paper is partially based on observations obtained with the Apache Point Observatory 3.5-meter telescope, which is owned and operated by the Astrophysical Research Consortium. We thank Bruce Elmegreen, Hans Zinnecker, and Bo Reipurth for their insights and comments. We thank the referee, Paul Ho, for a thorough reading and very helpful criticism that greatly improved the manuscript.

\section{REFERENCES}

Aarseth, S. J., Anosova, J. P., Orlov, V. V., \& Szebehely, V. G. 1994, Celest. Mech., 60, 131

Allen, D.A., \& Burton, M.G. 1993, Nature, 363, 54

Anosova, J. P. 1986, Ap\&SS, 124, 217

Arce, H. G., Shepherd, D., Gueth, F., Lee, C.-F., Bachiller, R., Rosen, A., \& Beuther, H. 2007, Protostars and Planets V, 245

Artymowicz, P., \& Lubow, S. H. 1994, ApJ, 421, 651

Axon, D. J., \& Taylor, K. 1984, MNRAS, 207, 241

Bally, J., \& Zinnecker, H. 2005, AJ, 129, 2281

Bally, J. 2008, in Massive Star Formation: Observations Confront Theory, ASPC, 387, 158, ed. H. Beuther

Baumgardt, H., \& Klessen, R. S. 2010, arXiv:1009.1189

Becklin, E.E. \& Neugebauer, G. 1967, ApJ, 147, 799

Beuther, H., \& Shepherd, D. 2005, Cores to Clusters: Star Formation with Next Generation Telescopes, 105

Beuther, H., \& Nissen, H. D. 2008, ApJ, 679, L121

Beuther, H., et al. 2006, ApJ, 636, 323

Bonnell, I. A., Bate, M. R., Clarke, C. J., \& Pringle, J. E. 2001, MNRAS, 323, 785

Cunningham, N. J. 2006, PhD thesis, University of Colorado, Boulder

Cunningham, N. J., Moeckel, N., \& Bally, J. 2009, ApJ, 692, 943 
Currie, D. G., Dowling, D. M., Shaya, E. J., Hester, J., Scowen, P., Groth, E. J., Lynds, R., O’neil, E. J., \& the Wide Field/Planetary Camera Instrument Definition Team 1996, AJ, 112, 1115

Cyganowski, C. J., et al. 2008, AJ, 136, 2391

Doi, T., ODell, C.R., \& Hartigan, P. 2002, AJ, 124, 445

Fryxell, B. A., \& Taam, R. E. 1988, ApJ, 335, 862

Furuya, R. S. , \& Shinnaga, H. 2009, arXiv:0908.0317v1

Genzel, R., Reid, M.J., Moran, J.M., \& Downes, D. 1981, ApJ, 244, 884

Gies, D. R. 1987, ApJS, 64, 545

Gies, D. R., \& Bolton, C. T. 1986, ApJS, 61, 419

Gezari, D. Y., Backman, D. E., \& Werner, M. W. 1998, ApJ, 509, 283

Goddi, C., Greenhill, L. J., Chandler, C. J., Humphreys, E. M. L., Matthews, L. D., \& Gray, M. D. 2009, ApJ, 698, 1165

Gomez, L., Rodriguez, L.F., Loinard, L., Lizano, S., Poveda, A., \& Allen, C. 2005, ApJ, 635, 1166

Gómez, L., Rodríguez, L. F., Loinard, L., Lizano, S., Allen, C., Poveda, A., \& Menten, K. M. 2008, ApJ, 685, 333

Greenhill, L.J., Gwinn, C.R., Schwartz, C., Moran, J.M., \& Diamond, P.J. 1998, Nature, 396,650

Grosso, N., Feigelson, E. D., Getman, K. V., Kastner, J. H., Bally, J., \& McCaughrean, M. J. 2006, A\&A, 448, L29

Gualandris, A., Portegies Zwart, S., \& Eggleton, P. P. 2004, MNRAS, 350, 615

Günther, R., \& Kley, W. 2002, A\&A, 387, 550

Gvaramadze, V. V. \& Gualandris, A. 2010, MNRAS (in press), arXiv:1007.5057v1

Hoogerwerf, R., de Bruijne, J. H. J., \& de Zeeuw, P. T. 2001, A\&A, 365, 49

Jiang, Z., Tamura, M., Fukagawa, M., Hough, J., Lucas, P., Suto, H., Ishii, M., \& Yang, J. 2005, Nature, 437, 112 
Johnstone, D., \& Bally, J. 1999, ApJ, 510, L49

Kaifu, N., et al. 2000, PASJ, 52, 1

Käpylä, P. J., Korpi, M. J., \& Brandenburg, A. 2008, A\&A, 491, 353

Kleinmann, D.E. \& Low, F. 1967, ApJ, 149, L1 Discovery of an infrared nebula in Orion.

Krumholz, M. R., McKee, C. F., \& Klein, R. I. 2005, ApJ, 618, 757

Krumholz, M. R., McKee, C. F., \& Klein, R. I. 2006, ApJ, 638, 369

Krumholz, M. R. 2006, ApJ, 641, L45

Kwan, J., \& Scoville, N.Z. 1976, ApJ, 210, L39

Lee, J.-K., \& Burton, M. G. 2000, MNRAS, 315, 11

Lubow, S. H. \& Artymowicz, P. 1996, ApJL, 467, 77

Lynden-Bell, D. 1996, MNRAS, 279, 389

Matthews, L. D., Goddi, C., Greenhill, L. J., Chandler, C. J., Reid, M. ., \& Humphreys, E.M.L. 2008, arXiv:0705.0699

Matt, S., Frank, A., \& Blackman, E. G. 2004, The Last Hurrah: PPN Formation by a Magnetic Explosion. ASP Conf. Ser. 313: Asymmetrical Planetary Nebulae III: Winds, Structure, and the Thunderbird, 313, 449

Matt, S., Frank, A., \& Blackman, E. G. 2006, ApJ, 647, L45

McCaughrean, M.J. \& Mac Low, M. 1997, AJ, 113, 391

McKee, C. F., \& Tan, J. C. 2002, Nature, 416, 59

McKee, C. F., \& Tan, J. C. 2003, ApJ, 585, 850

Menten, K. M. \& Reid, M. J. 1995, ApJ, 445, L157

Moeckel, N., \& Bally, J. 2007a, ApJ, 661, L183

Moeckel, N., \& Bally, J. 2007b, ApJ, 656, 275

Moeckel, N., \& Bally, J. 2006, ApJ, 653, 437

Moeckel, N. \& Throop, H. B. 2009, ApJ, 707, 268 
Moeckel, N., \& Clarke, C. J. 2010, arXiv:1009.0283

O’Dell, C. R., Hartigan, P., Bally, J., \& Morse, J. A. 1997, AJ, 114, 2016

Poveda A., Ruiz J., Allen C., 1967, Bul. Obs. Tonantzinla Tacubaya, 28, 86

Pontoppidan, K. M., \& Dullemond, C. P. 2005, A\&A, 435, 595

Plambeck, R. L., et al. 2009, ApJ, 704, L25

Poveda A., Ruiz J., \& Allen C. 1967. Bol. Obs. Tonantzintla Tacubaya 4, 86

Prince P.J. \& Dormand J. R. 1981, JCAM, 7, 67

Qiu, K., et al. 2008, ApJ, 685, 1005

Reid, M. J., Menten, K. M., Greenhill, L. J., \& Chandler, C. J. 2007, ApJ, 664, 950

Reipurth, B. 2000, AJ, 120, 3177

Reipurth, B., \& Bally, J. 2001, Ann. Rev. Astron. Astrophys., 39, 403

Rodriguez, L.F., Poveda, A., Lizano, S., \& Allen, C. 2005, ApJ, 627, L65

Rodriguez, L. F., Zapata, L. A., \& Ho, P. T. P. 2008 arXiv:0810.5055

Ruffert, M. 1997, A\&A, 317, 793

Ruffert, M. 1999, A\&A, 346, 861

Sahai, R., Claussen, M., Sánchez Contreras, C., Morris, M., \& Sarkar, G. 2008, ApJ, 680, 483

Schilbach, E., \& Roser, S. 2008, A\&A, 489, 105

Scoville, N.Z., Hall, D.N.B., Kleinmann, S.G. \& Ridgway, S.T. 1983, ApJ, 275, 201

Shima, E., Matsuda, T., Anzer, U., Boerner, G., and Boffin, H. M. J.: 1998, A\&A 337, 311

Shu, F. H., Lizano, S., Galli, D., Cai, M. J., \& Mohanty, S. 2008, ApJ, 682, L121

Shuping, R. Y., Morris, M., \& Bally, J. 2004, AJ, 128, 363

Simpson, J. P., Colgan, S. W. J., Erickson, E. F., Burton, M. G., \& Schultz, A. S. B. 2006, ApJ, 642, 339 
Smith, N., Whitney, B. A., Conti, P. S., de Pree, C. G., \& Jackson, J. M. 2009, MNRAS, 399, 952

Snell, R. L., Scoville, N. Z., Sanders, D. B., \& Erickson, N. R. 1984, ApJ, 284, 176

Sterzik, M. F., \& Durisen, R. H. 1995, A\&A, 304, L9

Sterzik, M. F. \& Durisen, R. H., 1998, A\&A, 339, 95

Stone, J.M., Xu, J., \& Mundy, L.G. 1995, Nature, 377, 315

Stone, R. C. 1991, AJ, 102, 333

Throop, H. B., \& Bally, J. 2008, AJ, 135, 2380

van Albada, T. S. 1968, Bull.Astron.Inst.Netherlands, 19, 479

Vasil, G. M., \& Brummell, N. H. 2009, ApJ, 690, 783

Vasil, G. M., \& Brummell, N. H. 2008, ApJ, 686, 709

Valtonen, M., \& Mikkola, S. 1991, ARA\&A, 29, 9

Wheeler, J.C. Meier, D.L., \& Wilson, J.R. 2002, ApJ, 568, 807

Wiseman, J.J., \& Ho, P.T.P. 1996, Nature, 382, 139

Wright, M. C. H., Plambeck, R. L., Mundy, L. G., \& Looney, L. W. 1995, ApJ, 455, L185

Zapata, L. A., Schmid-Burgk, J., Ho, P. T. P., Rodríguez, L. F., \& Menten, K. M. 2009, ApJ, 704, L45

Zapata, L. A., Schmid-Burgk, J., \& Menten, K. M. 2010, arXiv:1009.1426

Zinnecker, H. 1982, Annals of the New York Academy of Sciences , 395, 226

Zinnecker, H. \& Yorke, H. W. 2007, ARA\&A, 45, 481 


\section{Appendix 1: Excess Kinetic Energy of a Power-law Envelope After Ejection of an Interior Cluster.}

Assume that a gaseous envelope surrounds a compact cluster of stars with mass $M_{c l}$, and that the motion of the gas is dominated by gravitational forces. The stars are all interior to the inner spherical boundary at radius $r_{i n}$, and the average gas density is given by a power law so that $\rho(r)=\rho_{0} r^{-\alpha}$ out to an exterior radius $r_{\text {out }}$. If the total mass of the envelope is $M_{e}$, the normalization constant is

$$
\rho_{0}=\frac{(3-\alpha) M_{e}}{4 \pi\left(r_{\text {out }}^{3-\alpha}-r_{\text {in }}^{3-\alpha}\right)}
$$

The potential energy of the envelope is found from

$$
\begin{aligned}
W_{e} & =-4 \pi G \int_{0}^{\infty} r \rho(r) M_{t o t}(r) d r \\
& =-4 \pi G \int_{r_{\text {in }}}^{r_{\text {out }}} \rho_{0} r^{1-\alpha} M_{c l} \mathrm{~d} r-4 \pi G \int_{r_{\text {in }}}^{r_{\text {out }}} \rho_{0} r^{1-\alpha} M_{e}(r) \mathrm{d} r \quad(A 1-1) .
\end{aligned}
$$

where $M_{t o t}=M_{c l}+M_{e}(r)$ is the total mass inside radius $r$ before ejection of the cluster of massive stars. The first term is associated with the central cluster mass, while the second term is the potential associated with the gas envelope itself $M_{e}(r)$ being the envelope mass inside radius (r). Taking the pre-stellar-ejection envelope to be virialized, we have $-W_{\text {init }}=$ $2 T_{\text {init }}$. Because the observed stellar ejection velocities are much faster than the Kepler velocities in the envelope, stellar ejection happens on a timescale fast compared to the speed at which the gas can redistribute itself. Immediately after ejection of the stars, the potential energy of the envelope will be given solely by the second term, which remains the same, and the kinetic energy will still be given by $T_{\text {init }}$. If the post-ejection envelope were virialized, it would have $-W_{\text {final }}=2 T_{\text {final,vir }}$; the excess kinetic energy is then given by

$$
T_{\text {excess }}=T_{\text {init }}-T_{\text {final,vir }}=\left(-W_{\text {init }}+W_{\text {final }}\right) / 2=|\Delta W| / 2 .
$$

The quantity $|\Delta W|$ is simply the term in equation (A1-1) associated with the cluster mass, which integrates to give

$$
T_{\text {excess }}=\frac{G M_{c l} M_{e}}{2} \frac{(3-\alpha)}{(2-\alpha)}\left[\frac{r_{\text {out }}^{2-\alpha}-r_{\text {in }}^{2-\alpha}}{r_{\text {out }}^{3-\alpha}-r_{\text {in }}^{3-\alpha}}\right] \quad(A 1-2) .
$$




\section{Appendix 2: The Stored Kinetic Energy of an Idealized Power-law Disk}

To estimate the kinetic energy stored in the gravitationally bound motions of a circumstellar disks surrounding a star of mass $M_{\star}$, assume that between the inner edge of the disk, $r_{d, i n}$, and the outer edge at radius $r_{d, \text { out }}$, the average gas surface density is represented by a power-law on the radius of the form $\Sigma(r)=\Sigma_{0} r^{-\beta}$. With a total disk mass $M_{d}$, the normalization is given by

$$
\Sigma_{0}=\frac{(2-\beta) M_{d}}{2 \pi\left(r_{d, \text { out }}^{2-\beta}-r_{d, \text { in }}^{2-\beta}\right)}
$$

Assuming a disk in near-Keplerian rotation (thus with a disk mass small compared to the stellar mass), the potential energy of the system is to a good approximation just due to the stellar potential,

$$
W_{d} \approx-2 \pi G \int_{r_{d, \text { in }}}^{r_{d, \text { out }}} \Sigma_{0} r^{-\beta} M_{\star} \mathrm{d} r
$$

With Keplerian motion we have $-W_{d, \text { init }}=2 T_{d \text {,init }}$. Immediately after ejection of the star, which we assume happens on a timescale fast compared to the gas redistribution timescale, the potential energy of the disk will be due only to its self-potential, which we have neglected due to its assumed small contribution to the total. The excess kinetic energy is thus

$$
T_{d, \text { excess }}=T_{d, \text { init }}-T_{d, \text { final }, \text { vir }}=\left(-W_{d, \text { init }}+W_{d, \text { final }}\right) / 2=\left|\Delta W_{d}\right| / 2 .
$$

Since the stellar potential was dominant, the quantity $\left|\Delta W_{d}\right|$ is simply the potential energy due to the star from equation 1, which integrates out to

$$
T_{d, \text { excess }}=\frac{G M_{\star} M_{d}}{2} \frac{(2-\beta)}{(1-\beta)}\left[\frac{r_{d, \text { out }}^{1-\beta}-r_{d, \text { in }}^{1-\beta}}{r_{d, \text { out }}^{2-\beta}-r_{d, \text { in }}^{2-\beta}}\right] \quad(A 2-3) .
$$




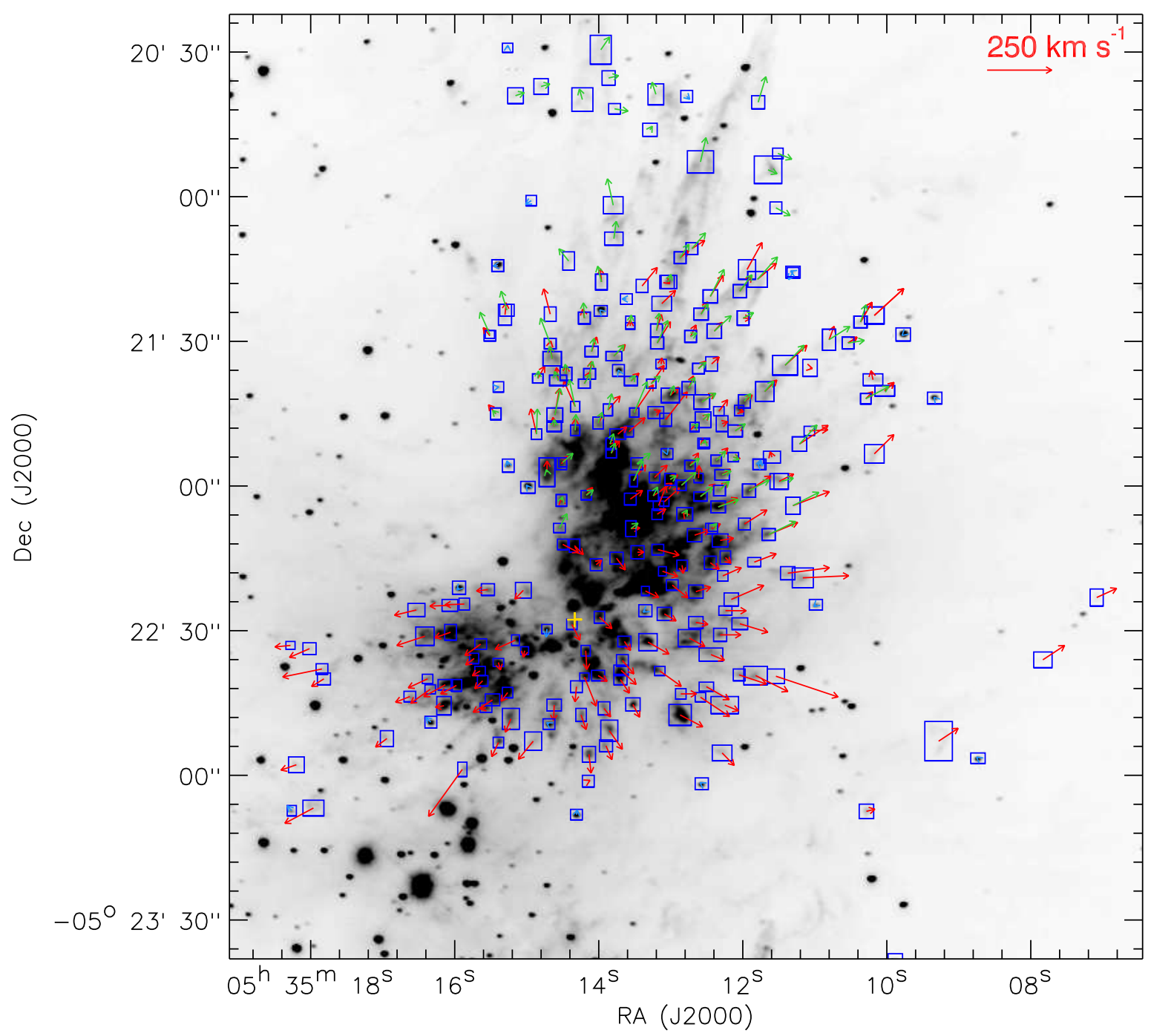

Fig. 1.- BN/KL outflow imaged in $\mathrm{H}_{2} 2.12 \mu \mathrm{m}$ emission in December 2004, from Cunningham (2006). Blue boxes indicate features with proper motion measurements. Green arrows denote proper motion measurements based on comparison of the 2004 data with prior-epoch images from September 1992 (Allen \& Burton 1993). Red arrows denote motions measured using January 1999 Subaru images (Kaifu et al. 2000). Short blue arrows indicate proper motion measurements on stars in the field, and verify proper image registration. Arrow lengths indicate velocities according to the scale at upper right, and are equivalent to 122-year motions. The yellow cross indicates the apparent point of origin of three ejected, massive stars, radio sources BN, I, and n (Gomez et al. 2005, 2008). 


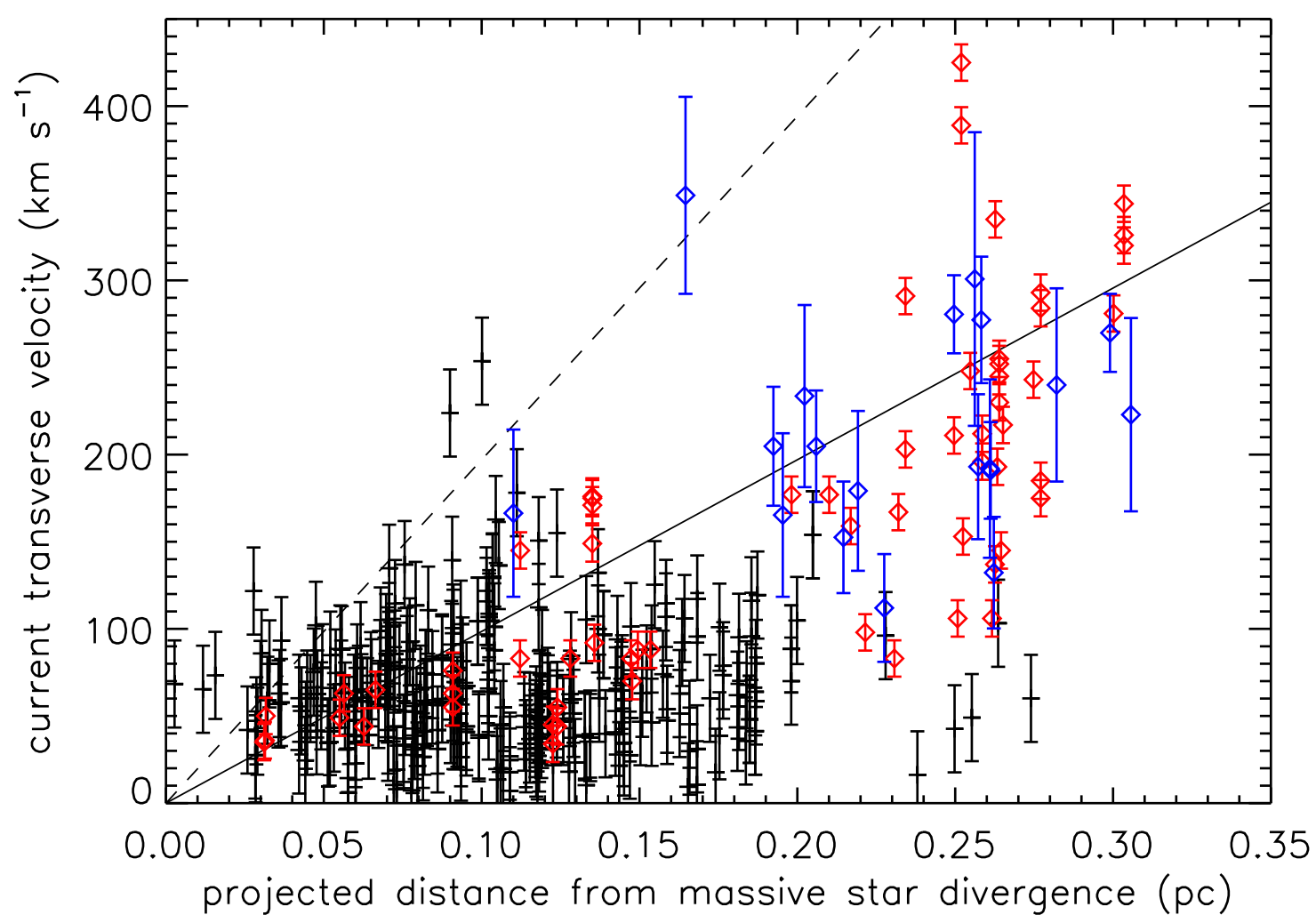

Fig. 2.- Projected distance versus transverse velocity for features in BN/KL with measured proper motions. Distances are measured relative to the center of divergence of the massive stars as reported by Gomez et al. (2005, 2008). Features and proper motions are optical HH objects measured by Doi, O’Dell, \& Hartigan (2002, red diamonds), [Fe II] 1.64 micron bullets measured by Lee \& Burton (2000, blue diamonds), and $\mathrm{H}_{2} 2.12 \mu \mathrm{m}$ features measured by Cunningham (2006, black crosses). The solid line indicates a zero-deceleration track for features launched 1000 years ago from the center of the dissociated system, and the dashed line indicates 500 year ages with no deceleration. The presence of many data points above the 1000 year track, together with the expectation of deceleration for these knots as they lose momentum and energy via interactions with the ambient medium, suggests an age younger than the typically-reported $\sim 1000$ years, and more consistent with the 500 year age of the multiple system dissociation event. 


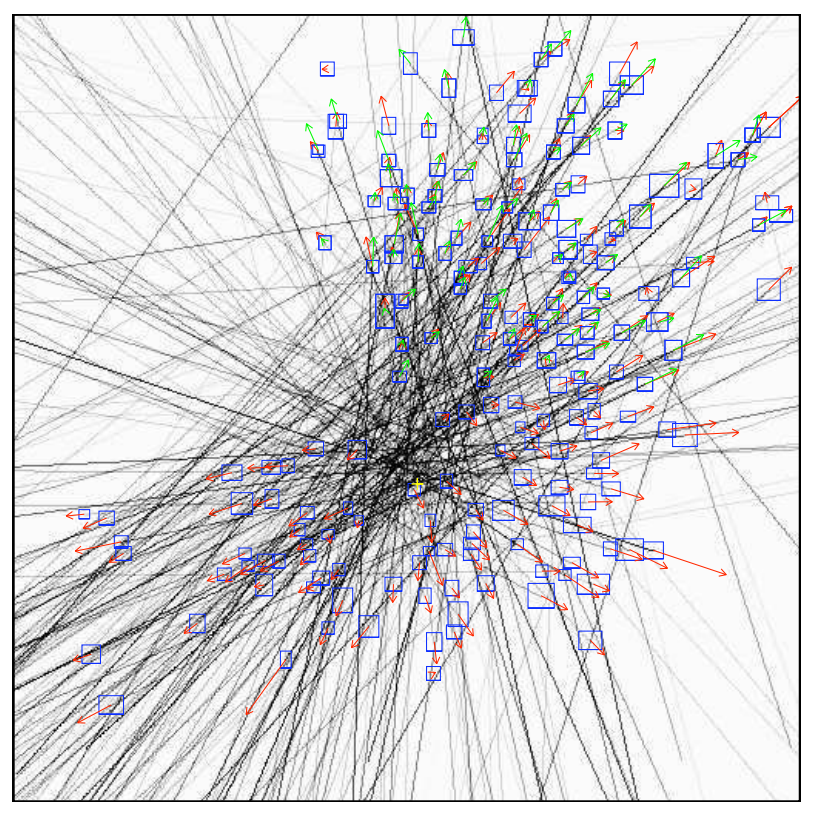

(a) Weighted, no smoothing.

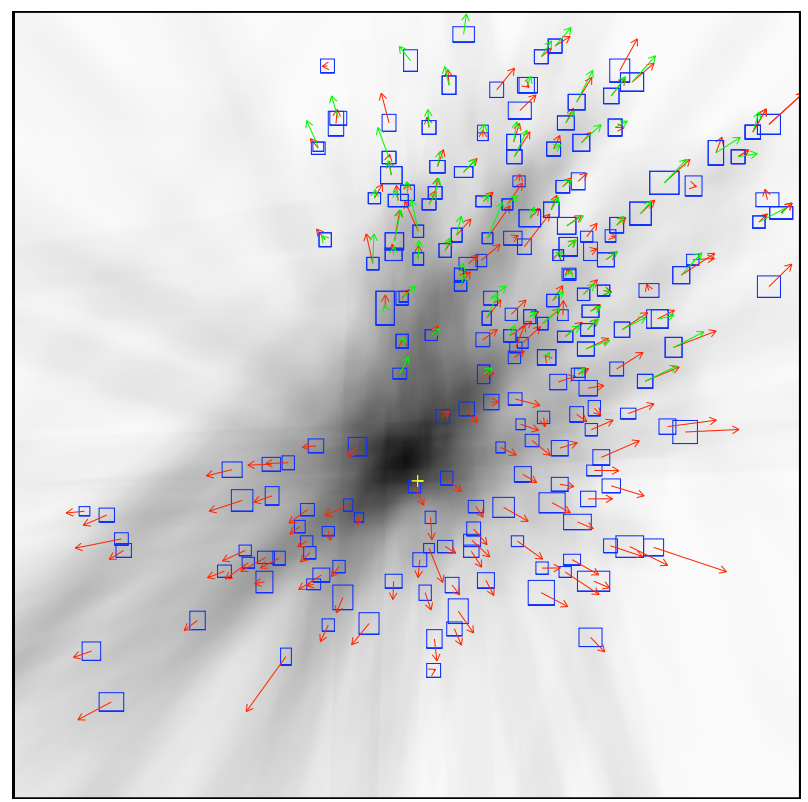

(b) Weighted, 31 pixel smoothing.

Fig. 3.- Results of the "trail" analysis to determine the point of origin of the proper motions motions in the $\mathrm{BN} / \mathrm{KL}$ outflow. The rays in the panels have been weighted by the magnitude of the associated velocity vector. The image in panel b has been boxcar smoothed by 31 pixels. The white cross marks the ejection point of radio sources BN, I, and n. Each frame is $150^{\prime \prime}$ in extent in the east-west and north-south directions. 


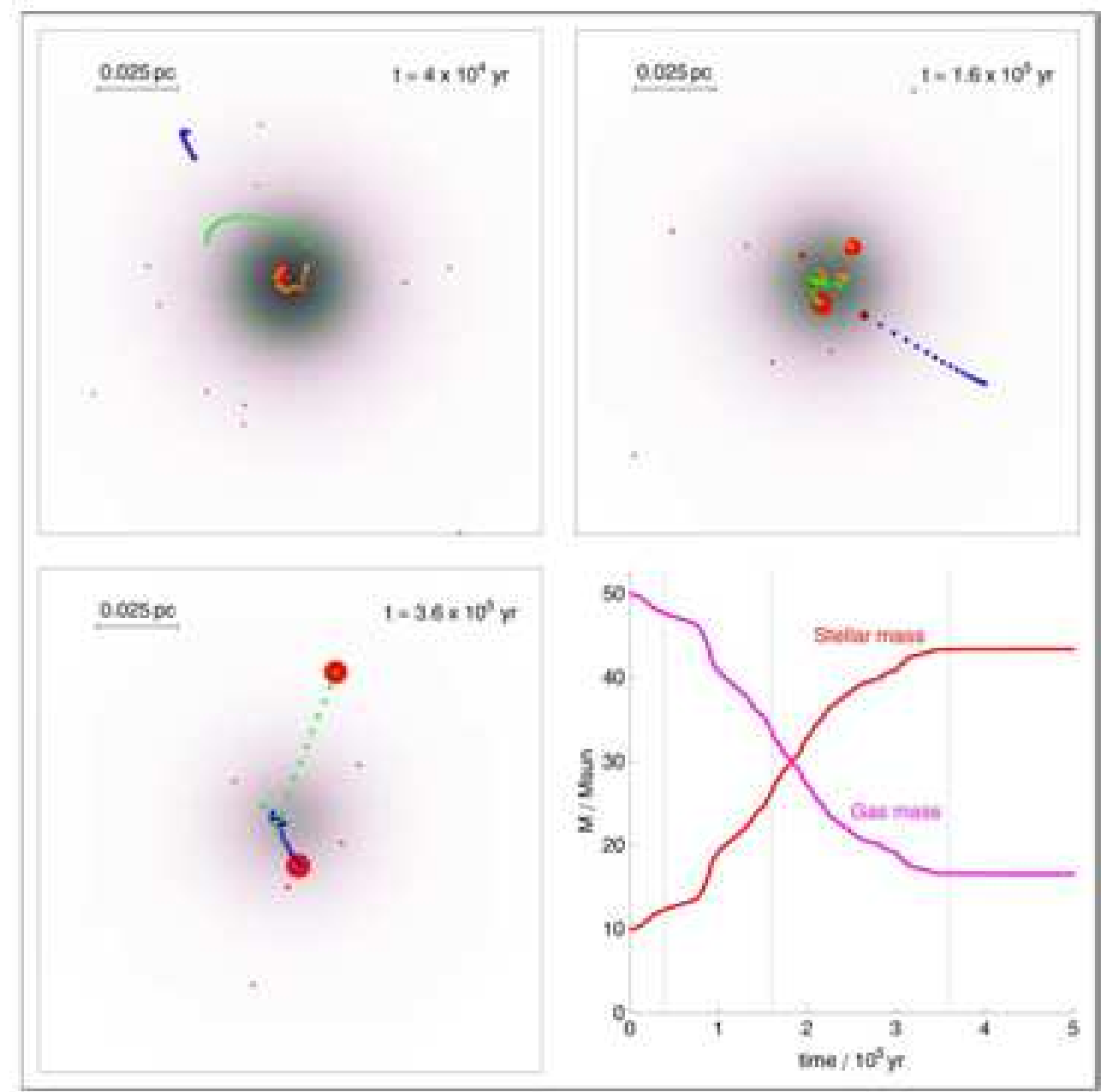

Fig. 4.- Four frames from a simulation of the formation of a non-hierarchcal multiple system of massive stars from a spherical clump of gas. The simulation starts with $50 \mathrm{M}_{\odot}$ of gas in a Plummer sphere with a core radius of 0.025 pc. 20 stellar seeds, each having an initial mass of $0.5 \mathrm{M}_{\odot}$ were uniformly distributed in a sphere with radius $0.1 \mathrm{pc}$, with virialized velocities. Seeds having the lowest speeds and those closest to the core experience the most rapid growth due to Bondi-Hoyle accretion. The orbits of these stars decay as they gain mass, eventually forming a non-hierarchical group near the center of the clump. Their total mass comes to dominate the gravitational potential and the stars are eventually dynamically ejected. The fourth panel shows the mass in gas and the mass in stars, with the times of the snapshots marked with vertical lines. The positions of stars involved in the final encounter that resulted in ejection during the last 20 time-steps are shown as colored trails. 


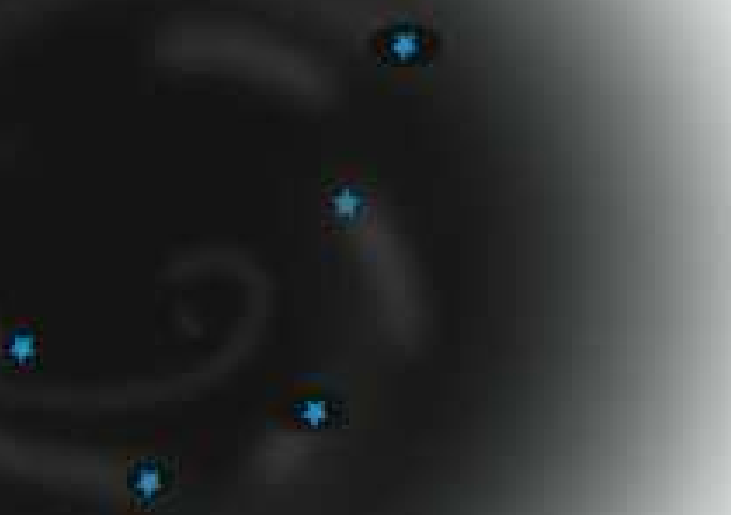

\section{Before formation of non-hlerarchical multiple of massive stars}

Fig. 5.- An illustration showing three stages in the evolution of $\mathrm{OMC} 1$ in the proposed scenario. (a): OMC1 before the formation of a massive non-hierarchical multiple system corresponding to the upper-left panel in Figure 4. (b): OMC1 after the formation of a massive non-hierarchical multiple system but before its decay corresponding to the upperright panel in Figure 4. (c): OMC1 following the ejection of massive stars corresponding to the lower-left panel in Figure 4. 


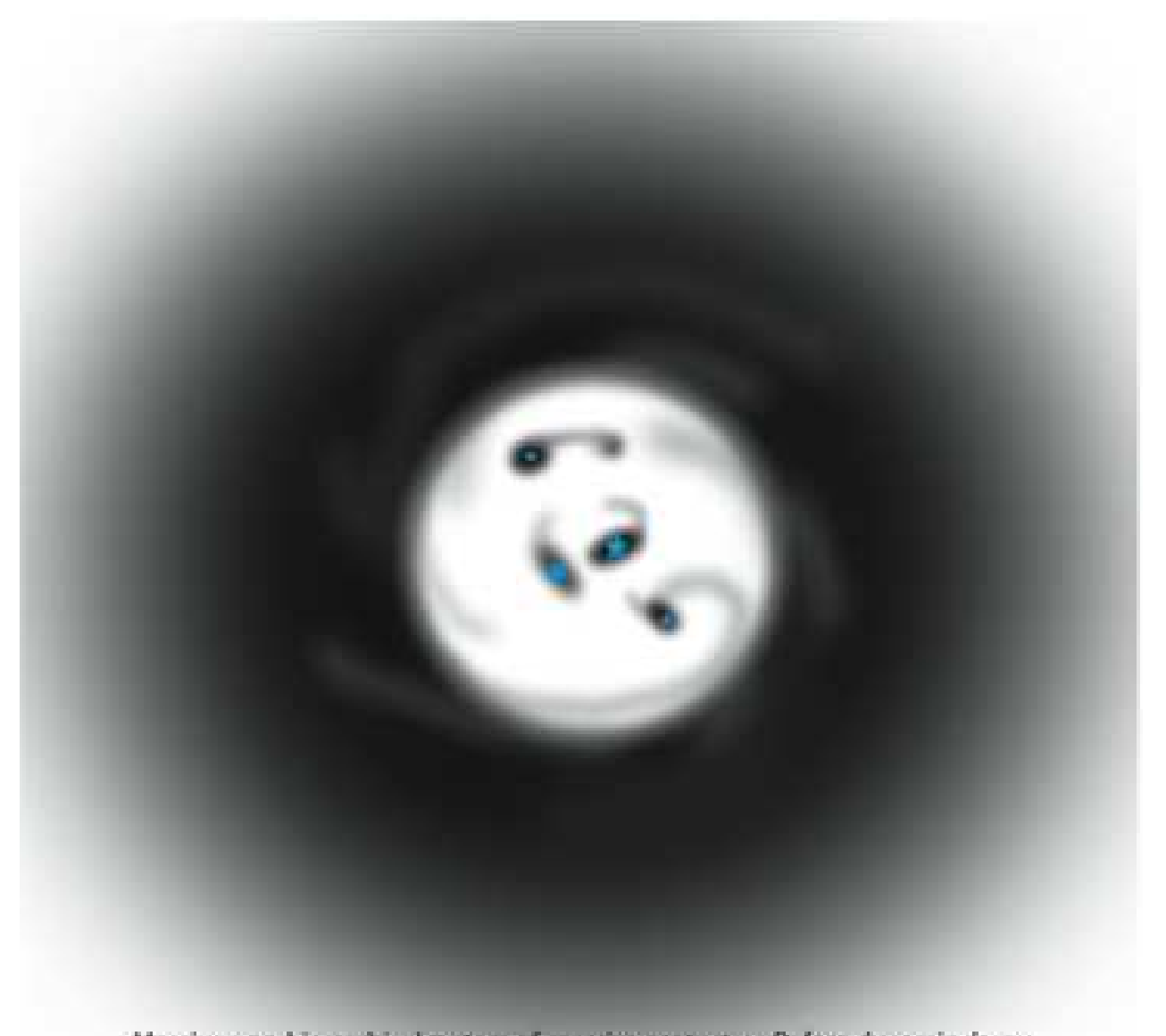

Massive non-hierachical system of massive protostars. Before dynamic decay 


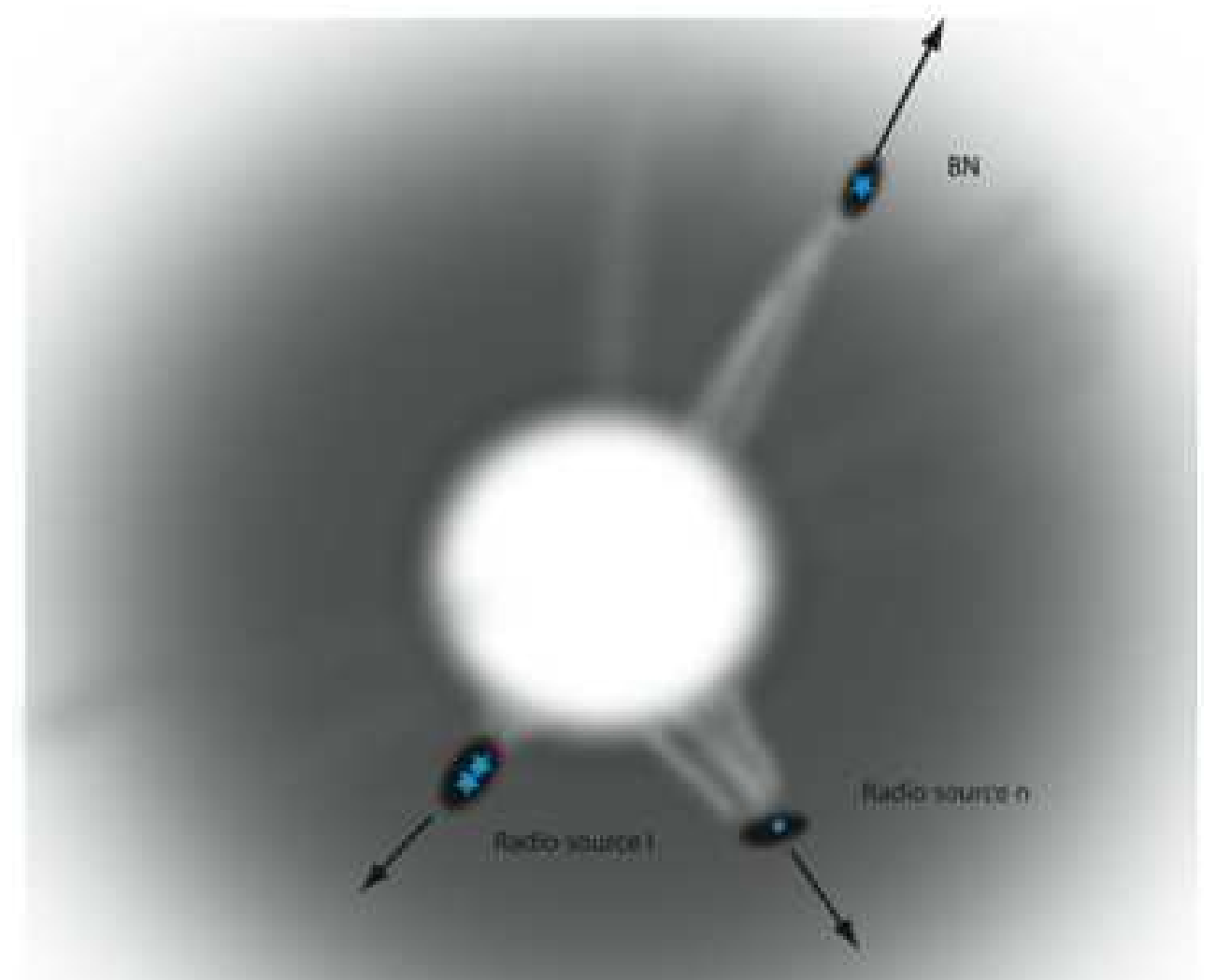

Following dynamic decay 
Table 1. Plausible Kinetic Energies of Ejected Envelopes

\begin{tabular}{cccccc}
\hline \hline$\alpha$ & $\begin{array}{c}R_{\text {out }} \\
(\mathrm{pc})\end{array}$ & $\begin{array}{c}R_{\text {in }} \\
(\mathrm{AU})\end{array}$ & $\begin{array}{c}M_{e} \\
\left(\mathrm{M}_{\odot}\right)\end{array}$ & $\begin{array}{c}M_{*} \\
\left(\mathrm{M}_{\odot}\right)\end{array}$ & $\begin{array}{c}E_{\text {excess }} \\
(\mathrm{ergs})\end{array}$ \\
\hline 1.5 & 0.1 & 300 & 60 & 40 & $2.7 \times 10^{45}$ \\
2.0 & 0.1 & 300 & 60 & 40 & $4.4 \times 10^{45}$ \\
1.5 & 0.1 & 50 & 60 & 40 & $2.9 \times 10^{45}$ \\
2.0 & 0.1 & 50 & 60 & 40 & $6.2 \times 10^{45}$ \\
1.5 & 0.1 & 300 & 60 & 20 & $1.3 \times 10^{45}$ \\
2.0 & 0.1 & 300 & 60 & 20 & $2.2 \times 10^{45}$ \\
1.5 & 0.1 & 50 & 60 & 20 & $1.5 \times 10^{45}$ \\
2.0 & 0.1 & 50 & 60 & 20 & $3.1 \times 10^{45}$ \\
\hline
\end{tabular}

Table 2. Plausible Kinetic Energies of Ejected Disks

\begin{tabular}{cccccc}
\hline \hline$\beta$ & $\begin{array}{c}R_{\text {out }} \\
(\mathrm{AU})\end{array}$ & $\begin{array}{c}R_{\text {in }} \\
(\mathrm{AU})\end{array}$ & $\begin{array}{c}M_{d} \\
\left(\mathrm{M}_{\odot}\right)\end{array}$ & $\begin{array}{c}M_{*} \\
\left(\mathrm{M}_{\odot}\right)\end{array}$ & $\begin{array}{c}E_{\text {excess }} \\
(\mathrm{ergs})\end{array}$ \\
\hline 1.5 & 10 & 0.5 & 0.5 & 10 & $1.9 \times 10^{46}$ \\
1.0 & 10 & 0.5 & 0.5 & 10 & $1.4 \times 10^{46}$ \\
0.5 & 10 & 0.5 & 0.5 & 10 & $1.0 \times 10^{46}$ \\
1.5 & 10 & 0.1 & 0.5 & 10 & $4.4 \times 10^{46}$ \\
1.0 & 10 & 0.1 & 0.5 & 10 & $2.0 \times 10^{46}$ \\
0.5 & 10 & 0.1 & 0.5 & 10 & $1.2 \times 10^{46}$ \\
1.5 & 10 & 0.05 & 0.5 & 10 & $6.2 \times 10^{46}$ \\
0.5 & 10 & 0.05 & 0.5 & 10 & $1.2 \times 10^{46}$ \\
1.5 & 10 & 0.05 & 1.0 & 10 & $1.3 \times 10^{47}$ \\
\hline
\end{tabular}

